\title{
BARYCENTER TECHNIQUE AND THE RIEMANN MAPPING PROBLEM OF CHERRIER-ESCOBAR
}

\author{
Martin Mayer \& Cheikh Birahim Ndiaye
}

\begin{abstract}
We solve in the affirmative the remaining cases of the Riemann mapping problem of Cherrier[35]-Escobar[38] first raised by Cherrier[35] in 1984. Indeed, performing a suitable scheme of the barycenter technique of Bahri-Coron[14] via the Almaraz[3]Chen[34]'s bubbles, we completely solve all the cases left open after the work of Chen[34]. Hence, combining our work with the ones of Almaraz[2], Chen[34], Cherrier[35], Escobar[38],[40] and Marques[55],[56], we have that every compact Riemannian manifold with boundary, of dimension greater or equal than three, and with finite Sobolev quotient, carries a conformal scalar flat metric with constant mean curvature on the boundary.
\end{abstract}

\section{Introduction and statement of the results}

In their attempt to suitably generalize the celebrated Riemann mapping theorem of Complex Analysis which asserts that every simply connected proper domain of the plane is conformally diffeomorphic to a disk, Cherrier[35]-Escobar[38] raised the question of whether every $n$ dimensional compact Riemannian manifold with boundary and $n \geq 3$ carries a conformal scalar flat Riemannian metric with constant mean curvature on the boundary. In [35], Cherrier gives a positive answer provided the curvatures are not too big in a reasonable geometric sense. In [38] and [40], Escobar provides a positive answer when $n=3$, $n=4$ or when $n=5$ and the boundary is umbilic and when $n \geq 6$ with the boundary being non-umbilic or the Riemannian manifold being locally conformally flat and the boundary being umbilic. Later, Marques[55],[56] gives a positive answer to some remaining cases, precisely when $n=4$ or 5 and the boundary is not umbilic, when $n \geq 8$ and the boundary is umbilic and not locally conformally flat with respect to the induced Riemannian metric, and when $n \geq 9$ with the boundary being umbilic and the Weyl tensor does not vanish identi-

Received June 15, 2015. 
cally on the boundary. In $[\mathbf{2}]$, Almaraz gives a positive answer when $n=6,7,8$, the boundary is umbilic and the Weyl tensor does not vanish identically on the boundary. Recently, Chen[34] solves the problem for many situations of the cases remaining after the above cited works and reduces the other ones to the positivity of the ADM mass of some class of asymptotically flat Riemannian manifolds, like she did in a joint work with S. Brendle for the boundary Yamabe problem in [23]. However, as in [23], the latter positivity is not known to hold for non-spin Riemannian manifolds of dimension greater or equal than eight.

Our main goal in this work is to use the algebraic topological argument of Bahri-Coron[14] to solve the cases left open by Almaraz[2], Chen[34], Cherrier[35], Escobar[38],[40] and Marques[55],[56], as we did in $[\mathbf{5 7}]$ for the boundary Yamabe problem to settle the cases remaining after the works of Escobar[37] and Brendle-Chen[23]. Indeed, performing a suitable scheme of the barycenter technique of BahriCoron[14] via the Almaraz[3]-Chen[34]'s bubbles, we prove a result for the Riemann mapping problem of Cherrier[35]-Escobar[38] which covers all the cases left open after the above cited works. We mention that there has been a lot of works about Yamabe and scalar curvature type problems for compact Riemannian manifolds with and without boundary, and their fully nonlinear and high-dimensional extensions, see, for example, $[\mathbf{1}],[\mathbf{4}],[\mathbf{5}],[\mathbf{6}],[\mathbf{7}],[\mathbf{8}],[\mathbf{1 0}],[\mathbf{1 1}],[\mathbf{1 3}],[\mathbf{1 4}],[\mathbf{1 5}],[\mathbf{1 6}],[\mathbf{1 8}]$, $[19],[20],[21],[22],[23],[24],[25],[26],[27],[28],[29],[30],[31]$, $[32],[33],[34],[36],[37],[38],[39],[41],[42],[43],[47],[48],[49]$, $[51],[52],[53],[54],[55],[56],[57],[58],[59],[60],[61],[62],[63]$, $[\mathbf{6 4}],[\mathbf{6 7}],[\mathbf{6 8}]$ and the references therein.

In order to state clearly our theorem, we first fix some notation. Given $(\bar{M}, g)$ a $n$-dimensional compact Riemannian manifold with boundary $\partial M$, interior $M$ and $n \geq 3$, we denote by $L_{g}=-4 \frac{n-1}{n-2} \Delta_{g}+R_{g}$ the conformal Laplacian of $(\bar{M}, g)$ and $B_{g}=\frac{4(n-1)}{n-2} \frac{\partial}{\partial n_{g}}+2(n-1) H_{g}$ the conformal Neumann operator of $(M, g)$, with $R_{g}$ denoting the scalar curvature of $(\bar{M}, g), \quad \Delta_{g}$ denoting the Laplace-Beltrami operator with respect to $g, H_{g}$ is the mean curvature of $\partial M$ in $(\bar{M}, g)$, namely $H_{g}:=\frac{1}{n-1} \operatorname{tr}_{\hat{g}} A_{g}$ where $A_{g}$ is the second fundamental form of $\partial M$ in $(\bar{M}, g)$ with respect to the inner normal direction, $\hat{g}:=\left.g\right|_{\partial M}$, $\frac{\partial}{\partial n_{g}}$ is the outer Neumann operator on $\partial M$ with respect to $g$. We remark that our $\left(L_{g}, B_{g}\right)$ is $\frac{4(n-1)}{n-2}$ times the one used in the work of Escobar[37] (see page 5). Furthermore, we define the following Escobar functional:

$$
\mathcal{E}_{g}(u):=\frac{\left\langle L_{g} u, u\right\rangle+\left\langle B_{g} u, u\right\rangle}{\left(\oint_{\partial M} u^{\frac{2(n-1)}{n-2}} d S_{g}\right)^{\frac{n-2}{n-1}}}, \quad u \in W_{+}^{1,2}(\bar{M}),
$$


where

$$
\left\langle L_{g} u, u\right\rangle:=\left\langle L_{g} u, u\right\rangle_{L^{2}(M)},\left\langle B_{g} u, u\right\rangle:=\left\langle B_{g} u, u\right\rangle_{L^{2}(\partial M)},
$$

$d S_{g}$ is the volume form with respect to the Riemannian metric induced by $g$ on $\partial M$ and

$$
W_{+}^{1,2}(\bar{M}):=\left\{u \in W^{1,2}(\bar{M}): u>0\right\},
$$

with $W^{1,2}(\bar{M})$ denoting the usual Sobolev space of functions which are $L^{2}$-integrable with their first derivatives (for more information, see $[\mathbf{9}]$ and $[46])$. Moreover, we recall that the Sobolev quotient of $(M, \partial M, g)$ is defined as

$$
\mathcal{Q}(M, \partial M, g):=\inf _{u \in W_{+}^{1,2}(\bar{M})} \mathcal{E}_{g}(u) .
$$

As in [57], here also we remind that the following conformally invariant set plays an important role in the existence part in [34]

$$
\mathcal{Z}_{g}:=\left\{x \in \partial M: \quad \limsup _{d_{g}(x, y) \longrightarrow 0}\left(d_{g}(x, y)\right)^{2-d}\left|W_{g}(y)\right|=0\right\},
$$

where $d_{g}$ and $W_{g}$ denote, respectively, the Riemannian distance and the Weyl tensor of $(\bar{M}, g)$ and $d:=\left[\frac{n-2}{2}\right]$ with $[\cdot]$ denoting the integer part function.

Now, having fixed the needed notation, we are ready to state our theorem which reads as follows:

Theorem 1.1. Assuming that $(\bar{M}, g)$ is a n-dimensional compact Riemannian manifold with boundary $\partial M$ and interior $M$ such that $\partial M$ is umbilic in $(\bar{M}, g), n \geq 6, \quad \mathcal{Q}(M, \partial M, g)>0$, and $\mathcal{Z}_{g}=\partial M$, then $(\bar{M}, g)$ carries a conformal scalar flat Riemannian metric with respect to which $\partial M$ has constant mean curvature.

Hence, since the only possible open cases for the Riemann mapping problem of Cherrier[35]-Escobar[38] are when the dimension of the manifold is greater or equal than 6 with umbilic boundary, positive Sobolev quotient and $\mathcal{Z}_{g}=\partial M$, then clearly Theorem 1.1 and the works of Almaraz[2], Chen[34], Cherrier[35], Escobar[38],[40] and Marques[55],[56] imply the following positive answer to the high-dimensional generalization by Cherrier[35]-Escobar[38] of the celebrated Riemann mapping problem of Complex Analysis.

Theorem 1.2. Every $n$-dimensional compact Riemannian manifold with boundary, $n \geq 3$, and finite Sobolev quotient, carries a conformal scalar flat Riemannian metric with constant mean curvature on the boundary.

To give a positive answer to the high-dimensional generalization by Cherrier[35]-Escobar[38] of the celebrated Riemann mapping problem of Riemann surface theory is equivalent to solving a second order elliptic boundary value problem with critical Sobolev nonlinearity on the 
boundary. Indeed, under the assumptions of Theorem 1.1, the Riemann mapping problem of Cherrier[35]-Escobar[38] is equivalent to finding a smooth and positive solution of the following semilinear elliptic boundary value problem:

$$
\left\{\begin{array}{l}
L_{g} u=0 \text { in } M, \\
B_{g} u=2(n-1) u^{\frac{n}{n-2}} \quad \text { on } \partial M .
\end{array}\right.
$$

The boundary value problem (2) has a variational structure. Indeed, thanks to the work of Cherrier[35], smooth solutions of (2) can be found by looking at critical points of the Escobar functional $\mathcal{E}_{g}$, and as in [57], we will pursue such an approach here. Precisely, we will perform a suitable application of the barycenter technique of Bahri-Coron[14] via the Almaraz $[\mathbf{3}]-$ Chen[34]'s bubbles. However, since the application of the barycenter technique that we are going to present in this work is similar to the one we perform in [57], and since the algebraic topological argument of Bahri-Coron[14] (see [44], [45], [62], [65] for some applications of it) seems to be less known ("mastered") in the Geometric Analysis community in comparison to his companion argument, namely the Aubin[8]-Schoen[66]'s minimization technique, then we feel more convenient to describe it more in details here, for those who are not familiar with. We are going to describe the barycenter technique of Bahri-Coron[14] focusing on $\mathcal{E}_{g}$. To begin, the algebraic topological argument of Bahri-Coron[14] belongs the class of indirect methods. Precisely, it is an argument by contradiction. Thus, assuming that the Euler-Lagrange functional $\mathcal{E}_{g}$ has no critical points, one looks for a contradiction by using the quantization and strong interaction phenomenon that $\mathcal{E}_{g}$ verifies and the cone structure of the space of barycenters of $\partial M$. To describe how the combination of the latter works to give the desired contradiction, we think it is for the sake of understanding of the reader more useful to do it with pictures and some talking mathematical formulas rather than only exact mathematical ones, of course at the price of precision, but with the right intuition of what is going on with the barycenter technique of Bahri-Coron[14]. First of all, and recalling the assumption $\mathcal{E}_{g}$ has no critical points (to keep in mind), one has that the quantization phenomenon that $\mathcal{E}_{g}$ enjoys implies Fig. 1, which traduces the fact that by bubbling $\partial M$ survives topologically between the first and second critical levels of $\mathcal{E}_{g}$ that we denote by $\left(W_{1}, W_{0}\right)$ (see $(7)$ for its precise definition). Next, realizing $B_{2}(\partial M)$ (for its definition see (19)) as a cone over $B_{1}(\partial M)=\partial M$ with top $\partial M$, one has that the quantization phenomenon that $\mathcal{E}_{g}$ satisfies implies again Fig. 2 which shows the fact that by bubbling $B_{2}(\partial M)$ as a cone over $B_{1}(\partial M)$ survives as a nontrivial cone between the second and third critical levels of $\mathcal{E}_{g}$ that we denote by $\left(W_{2}, W_{1}\right)$. Similarly, realizing $B_{3}(\partial M)$ as a cone 


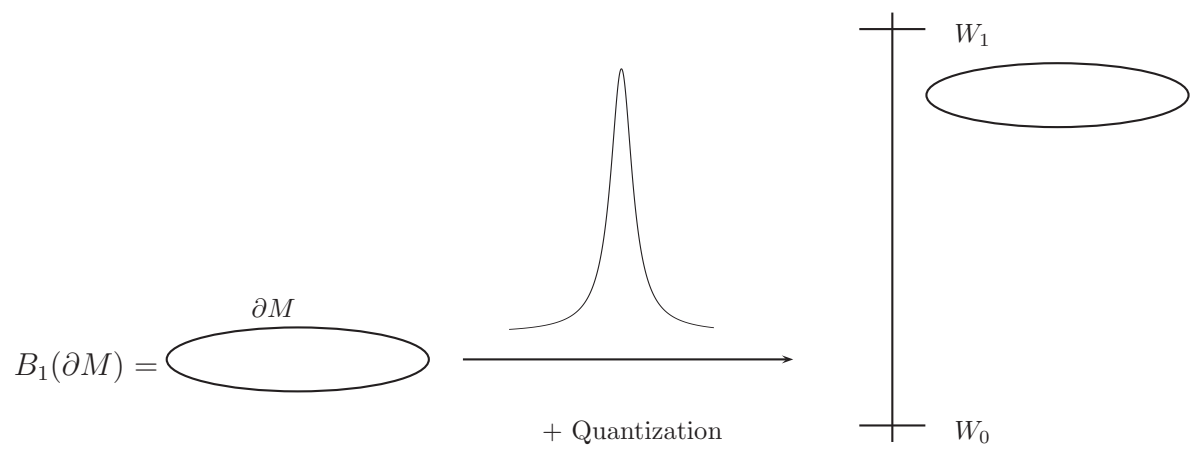

Figure 1

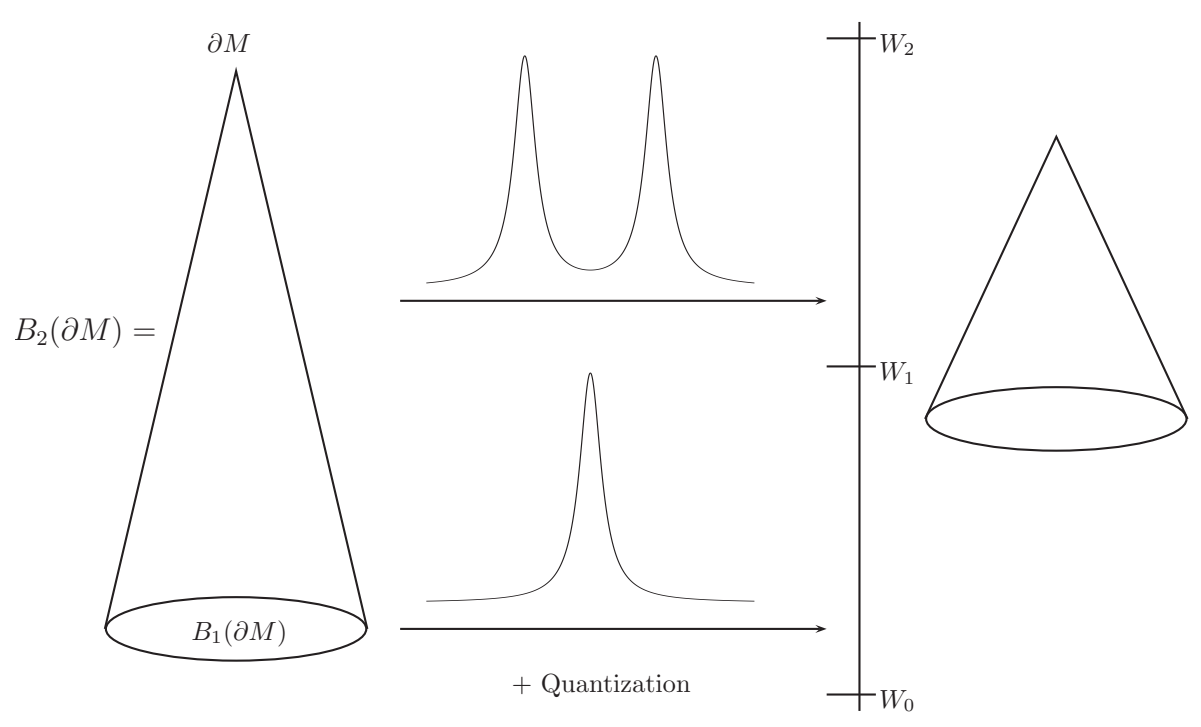

Figure 2

over $B_{2}(\partial M)$ with top $\partial M$, one has that the quantization phenomenon that $\mathcal{E}_{g}$ verifies implies again Fig. 3 which traduces the fact that by bubbling $B_{3}(\partial M)$ as a cone over $B_{2}(\partial M)$ survives as a nontrivial cone between the third and fourth critical levels of $\mathcal{E}_{g}$ that we denote by $\left(W_{3}, W_{2}\right)$. Hence, recursively for $p \in \mathbb{N}^{*}$, realizing $B_{p+1}(\partial M)$ as a cone over $B_{p}(\partial M)$ with top $\partial M$, we have that the quantization phenomenon that $\mathcal{E}_{g}$ enjoys implies again Fig. 4 which shows the fact that by bubbling $B_{p+1}(\partial M)$ as a cone over $B_{p}(\partial M)$ survives as a nontrivial cone between the $(p+1)$ and $(p+2)$ critical levels of $\mathcal{E}_{g}$ that we denote by $\left(W_{p+1}, W_{p}\right)$. On the other hand, the latter recursion leads to a contradiction because of the strong interaction phenomenon that $\mathcal{E}_{g}$ enjoys. To see this, one first observes that, as standard bubbles, the Almaraz $[\mathbf{3}]-$ Chen[34]'s bubbles $\varphi_{a, \lambda}$ verify the every where selfaction 


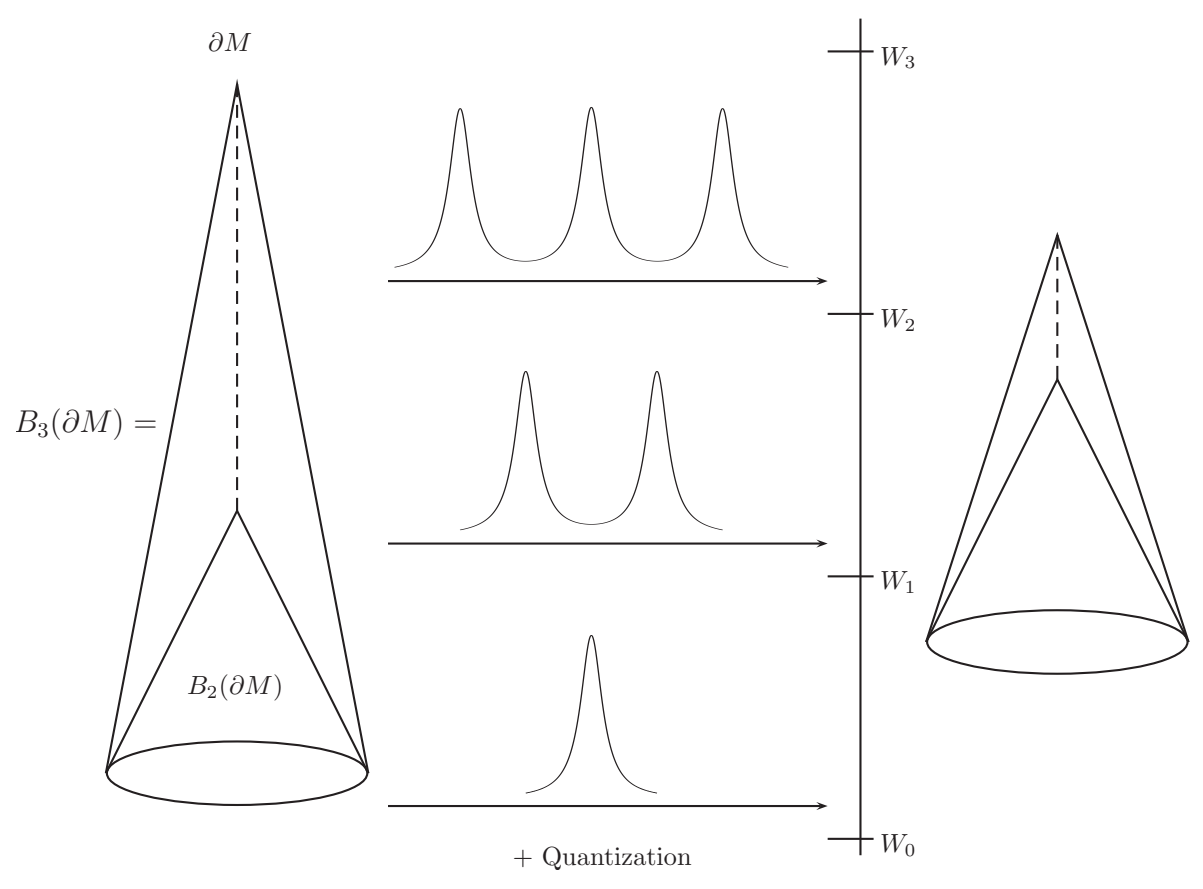

Figure 3

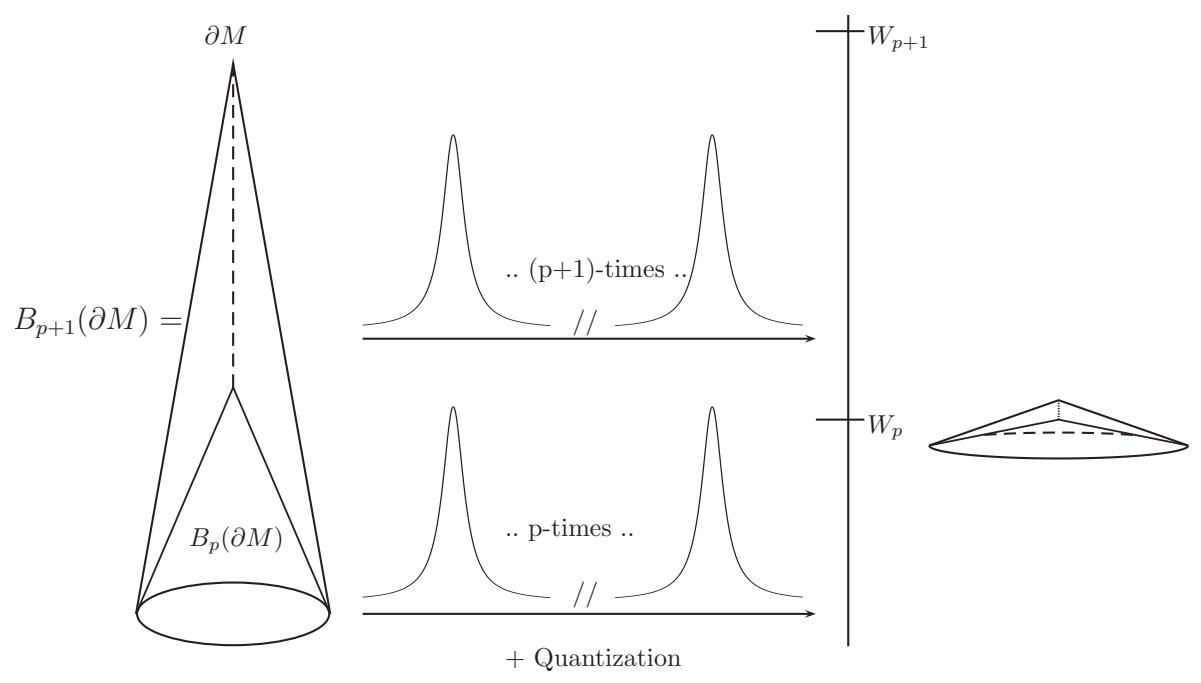

Figure 4

and interaction identities (see Lemma 2.8)

(3)

$$
\begin{aligned}
\left\langle L_{g} \varphi_{a_{i}, \lambda}, \varphi_{a_{j}, \lambda}\right\rangle & +\left\langle B_{g} \varphi_{a_{i}, \lambda}, \varphi_{a_{j}, \lambda}\right\rangle \\
= & (1+o(1))(n-2) \oint_{\partial M} \varphi_{a_{i}, \lambda}^{\frac{n}{n-2}} \varphi_{a_{j}, \lambda} d S_{g}, \quad a_{i}, a_{j} \in \partial M .
\end{aligned}
$$


Moreover, one observes also that, as standard bubbles, the Almaraz[3]Chen[34]'s bubbles $\varphi_{a, \lambda}$ verify the following interaction identity at infinity (see formula (18) and Lemma 2.8)

$$
\oint_{\partial M} \varphi_{a_{i}, \lambda}^{\frac{n}{n-2}} \varphi_{a_{j}, \lambda} d S_{g}=\frac{c_{n}+o(1)}{\left(1+\lambda^{2} G_{g}^{\frac{2}{2-n}}\left(a_{i}, a_{i}\right)\right)^{\frac{n-2}{2}}}, \quad a_{i} \neq a_{j} \in \partial M
$$

where $c_{n}$ is a positive constant pedending only on $n, G_{g}$ is the Green's function of $\left(L_{g}, B_{g}\right)$ suitably normalized, and $o(1)$ in the above formula means a quantity tending to 0 uniformly in $A:=\left(a_{i}, a_{j}\right)$ as $\lambda$ tends to $+\infty$. We would like to emphasize that, formula (3) for different particles $a_{i} \neq a_{j}$ is saying nothing else than, the linear interaction given by the right hand side of (3) is almost (up to the multiplication by $(n-2))$ the same as the nonlinear interaction given by the right hand side of (4), and that the value of the nonlinear interaction is almost $\frac{G_{g}\left(a_{i}, a_{j}\right)}{\lambda^{n-2}}$ (up to the multiplication by $c_{n}$ ). We would like to add also that the latter discussion reflects also nothing else that the restoration of symmetry, which is part of what defines infinity, because of the conformal invariance of the problem, and is consistent with invariance principles. Furthermore, for $p \in \mathbb{N}^{*},(3)$, (4) and the scale invariance of $\mathcal{I}_{g}$ imply that for optimal configurations $\sum_{i=1}^{p} \varphi_{a_{i}, \lambda}$ there holds

(5) $\mathcal{I}_{g}\left(\sum_{i=1}^{p} \varphi_{a_{i}, \lambda}\right) \leq \sum_{i=1}^{p}\left(\mathcal{E}_{g}\left(\varphi_{a_{i}, \lambda}\right)-(1+o(1)) c^{n} \sum_{j \neq i}^{p} \operatorname{Int}_{g}\left(a_{i}, a_{j}, \lambda\right)\right)$,

where $c^{n}$ is a positive constant depending only on $n, \operatorname{Int}_{g}\left(a_{i}, a_{j}, \lambda\right)$ denotes the interaction between $\varphi_{a_{i}, \lambda}$ and $\varphi_{a_{j}, \lambda}$ measured with respect to the left hand side of (3) or (4), and of course $c^{n}$ depends on which way one sees $\operatorname{Int}_{g}\left(a_{i}, a_{j}, \lambda\right)$. We would like to comment on the sign in formula (5) and say some words about optimal configurations. The latter sign does not come by miracle, but is part of what defines "true" infinity for the associated variational problem, and analytically, it can be seen just by Taylor expansion (which is possible because of being at infinity), and the fact that the scale invariance of $\mathcal{E}_{g}$ and (3) imply that, the denominator in the definition of $\mathcal{E}_{g}$ brings (roughly speaking) a contribution of -2 times the linear interaction in the calculation of $\mathcal{I}_{g}\left(\sum_{i=1}^{p} \varphi_{a_{i}, \lambda}\right)$, and the numerator brings a contribution of +1 times the linear interaction in the latter calculation. We have chosen to focus on optimal configurations to show how the strong interaction pushes the energy down for a large number of particles, this is due to the fact that those configurations are the most "variationally" critical ones, and to have a picture in mind, one can just think of the top of a mountain, or the down of a black hole. Now, an important piece in the argument is the fact that, the concentration points live in a 


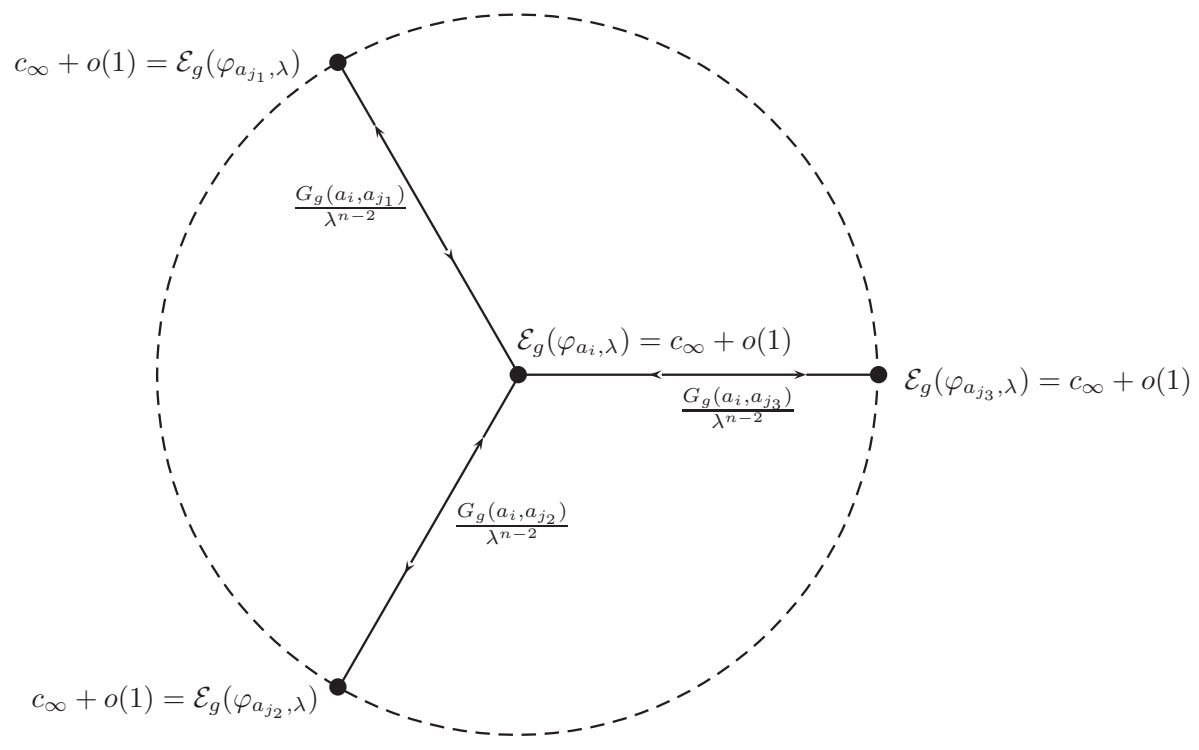

Figure 5

world with quantization and strong interaction phenomenon as shown by Fig. 5 where $c_{\infty}$ is the first critical value and that they verify the sharp mass energy estimate

$$
\mathcal{E}_{g}\left(\varphi_{a, \lambda}\right) \leq c_{\infty}-\frac{M D_{g}(a)}{\lambda^{n-2}}+o\left(\frac{1}{\lambda^{n-2}}\right),
$$

where $M D_{g}(a)$ denotes the mass distribution of the particle $a, o(1)$ means a quantity tending to 0 uniformly in $a$ as $\lambda$ tends to $+\infty$, and we recall that the interaction terms in the picture (5) are nothing else than a "representation" of $\operatorname{Int}_{g}\left(a_{i}, a_{j}, \lambda\right)$, namely

$$
\operatorname{Int}_{g}\left(a_{i}, a_{j}, \lambda\right)=\bar{c}_{n}(1+o(1)) \frac{G_{g}\left(a_{i}, a_{j}\right)}{\lambda^{n-2}},
$$

where $\bar{c}_{n}$ is a positive constant depending only on $n$, and still as above $\bar{c}_{n}$ depends on how one sees $\operatorname{Int}_{g}\left(a_{i}, a_{j}, \lambda\right)$ according to the left hand side of (3) and (4). Moreover, $M D_{g}$ is an $L^{\infty}$-function and $\min _{\bar{M}^{2}} G_{g}>0$ which is what we mean by $\mathcal{E}_{g}$ verifies a strong interaction phenomenon. We would like to remark the fact that in the pictures (1)-(4), we choose to emphasize that more the number of particles is large, less is the height of the cone, and this is to traduce the fact that (roughly speaking) for a very large number of particles in consideration, more is the number of particles, less is the "topological" space that we have to put the corresponding cone, as explained analytically by the formulas (5)-(6). Thus, clearly (5)-(6) imply Fig. 6 which shows the fact that for $p_{0}$ large, $B_{p_{0}}(\partial M)$ as a cone over 


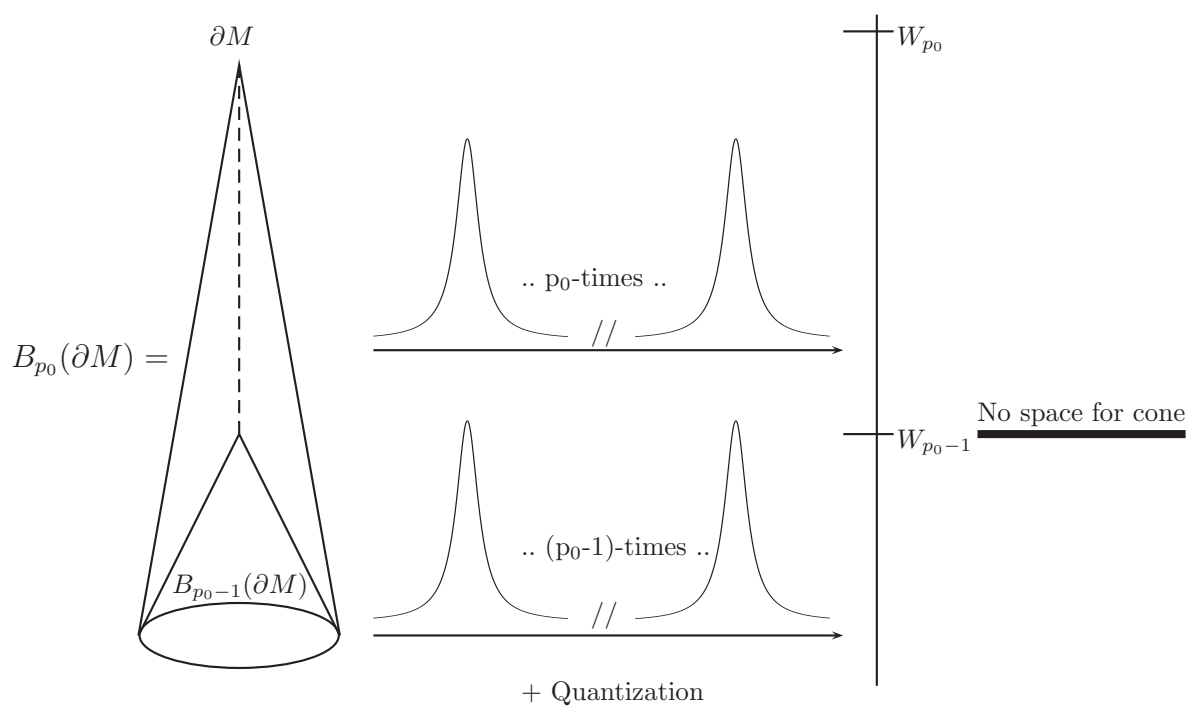

Figure 6

$B_{p_{0}-1}(\partial M)$ cannot be embedded by bubbling and still be a nontrivial cone between the $p_{0}$ and $p_{0}+1$ critical levels of $\mathcal{E}_{g}$. Hence, clearly the figures (4) and (6) lead to a contradiction.

The structure of this paper is as follows. In Section 2, we fix some notation and give some preliminaries, like the set of formal barycenters of $\partial M$ and present some useful topological properties of them. Furthermore, we recall the Almaraz $[\mathbf{3}]-$ Chen $[\mathbf{2 3}]$ 's bubbles and the fact that they can be used to replace the standard bubbles in the analysis of diverging Palais-Smale (PS) sequences of the Euler-Lagrange functional $\mathcal{E}_{g}$. Moreover, using a result of Almaraz[3] and another one of Chen $[\mathbf{3 4}]$ (or of Almaraz[3]), we derive selfaction and interaction estimates for the Almaraz[3]-Chen[34]'s bubbles. In Section 3, we use the latter estimates to map the space of barycenters of $\partial M$ of any order into suitable sublevels of $\mathcal{E}_{g}$ via the Almaraz $[\mathbf{3}]-$ Chen $[\mathbf{3 4}]$ 's bubbles. Finally, in Section 4, we define the neighborhood of potential critical points at infinity of $\mathcal{E}_{g}$ and use the results of Section 3 to carry our scheme of the barycenter technique of Bahri-Coron[14] to prove Theorem 1.1 .

Acknowledgments. C. B. Ndiaye has been supported by the DFG project "Fourth-order uniformization type theorems for 4-dimensional Riemannian manifolds". The authors would like to thank Pr. Mohameden Ahmedou, Pr. Abbas Bahri, Pr. Andrea Malchiodi and Pr. Reiner Michael Schaetzle for their interest in this work. The authors are also grateful to Pr. Emmanuel Hebey for comments about the work 
of Cherrier[35] and the referee for suggestions, which improve the presentation of the paper.

\section{Notation and preliminaries}

In this section, we fix some notations and give some preliminaries. First of all, since the problem under study is conformally invariant and we are dealing with the umbilic case, then from now until the end of the paper $(\bar{M}, g)$ will be the given underlying compact $n$-dimensional Riemannian manifold with boundary $\partial M$ and interior $M, \partial M$ is totally geodesic in $(\bar{M}, g), n \geq 6, \mathcal{Q}(M, \partial M, g)>0$, and $\mathcal{Z}_{g}=\partial M$.

In the following, for any Riemannian metric $\bar{g}$ on $\bar{M}$ and $p \in \partial M$, we will use the notation $B_{p}^{\bar{g}}(r)$ to denote the coordinate ball with respect to $\bar{g}$ of radius $r$ and center $p$. For $p \in \partial M$, we use the notation $\hat{B}_{p}^{\bar{g}}(r)$ to denote the geodesic ball in $\partial M$ with respect to the Riemannian metric $\hat{\bar{g}}$ induced by $\bar{g}$ on $\partial M$ of radius $r$ and center $p$. We also denote, respectively, by $d_{\bar{g}}(x, y)$ and $\bar{d}_{\bar{g}}(x, y)$, the geodesic distance with respect to $\bar{g}$ between two points $x$ and $y$ of $\bar{M}$, and the coordinate distance between $x \in \partial M$ and $y \in \bar{M}$. Similarly, we denote by $d_{\hat{\bar{g}}}(x, y)$, the geodesic distance with respect to $\hat{\bar{g}}$ between two points $x$ and $y$ of $\partial M \cdot \operatorname{inj}_{\bar{g}}(\bar{M}), i n j_{\hat{\bar{g}}}(\partial M)$ stand for the injectivity radius of $(\bar{M}, \bar{g}),(\partial M, \hat{\bar{g}}) . d V_{\bar{g}}$ denotes the Riemannian measure associated to the metric $\bar{g}$ and $d S_{\bar{g}}$ the volume form on $\partial M$ with respect to $\hat{\bar{g}}$ on $\partial M$. For simplicity, given $p \in \partial M$, we will use, respectively, $B_{p}(r)$ and $\hat{B}_{p}(r)$ to denote $B_{p}^{g}(r)$ and $\hat{B}_{p}^{g}(r)$. For $a \in \bar{M}$, we use the notation $\exp _{a}^{\bar{g}}$ to denote the exponential map with respect to $\bar{g}$ and set for simplicity $\exp _{a}:=\exp _{a}^{g}$. For $a \in \partial M$, we denote by $\exp _{a}^{\bar{g}}$ the exponential map with respect to $\hat{\bar{g}}$ and set $\exp _{a}:=\exp _{a}^{g}$.

$\mathbb{N}$ denotes the set of nonnegative integers, $\mathbb{N}^{*}$ stands for the set of positive integers and for $k \in \mathbb{N}^{*}, \mathbb{R}^{k}$ stands for the standard $k$-dimensional Euclidean space, $\mathbb{R}_{+}^{k}$ the open positive half-space of $\mathbb{R}^{k}$ and $\overline{\mathbb{R}}_{+}^{k}$ its closure in $\mathbb{R}^{k}$. For simplicity, we will use the notation $\mathbb{R}_{+}:=\mathbb{R}_{+}^{1}$ and $\overline{\mathbb{R}}_{+}:=\overline{\mathbb{R}}_{+}^{1}$. For $r>0, B_{0}^{\mathbb{R}^{k}}(r)$ denotes the open ball of $\mathbb{R}^{k}$ of center 0 and radius $r$ and set for simplicity $B^{k}:=B_{0}^{\mathbb{R}^{k}}(1)$. We use $g_{B^{k}}$ to denote the Euclidean metric on $B^{k}$. For $p \in \mathbb{N}^{*}, \sigma_{p}$ denotes the permutation group of $p$ elements, $(\partial M)^{p}$ denotes the Cartesian product of $p$ copies of $\partial M$. We define $\left((\partial M)^{2}\right)^{*}:=(\partial M)^{2} \backslash \operatorname{Diag}\left((\partial M)^{2}\right)$ where $\operatorname{Diag}\left((\partial M)^{2}\right)$ is the diagonal of $(\partial M)^{2}$, namely $\operatorname{Diag}\left((\partial M)^{2}\right):=$ $\{(a, a): a \in \partial M\}$. For $p \in \mathbb{N}^{*}, \Delta_{p-1}$ the following simplex $\Delta_{p-1}:=$ $\left\{\left(\alpha_{1}, \cdots, \alpha_{p}\right): \alpha_{i} \geq 0, i=1, \cdots, p, \sum_{i=1}^{p} \alpha_{i}=1\right\}$.

For $1 \leq p \leq \infty$ and $k \in \mathbb{N}, \beta \in] 0,1\left[, L^{p}(M)\right.$ and $L^{p}(\partial M)$, $W^{k, p}(M), C^{k}(\bar{M})$ and $C^{k, \beta}(\bar{M})$ stand, respectively, for the standard $p$-Lebesgue space on $M$ and $\partial M,(k, p)$-Sobolev space, $k$-continuously 
differentiable space and $k$-continuously differential space of Hölder exponent $\beta$, all with respect to $g$ (if the definition needs a metric structure) and for precise definitions and properties, see, for example, [9] or [46].

For $a \in \partial M, O_{a}(1)$ stands for quantities bounded uniformly in $a$. For $\epsilon$ positive and small and $a \in \partial M, O_{a, \epsilon}(1)$ stands for quantities uniformly bounded in $a$ and $\epsilon$. For $\epsilon$ positive and small, $o_{\epsilon}(1)$ means quantities which tend to 0 as $\epsilon$ tends to 0 . For $\lambda$ large and $a \in \partial M$, $O_{a, \lambda}(1)$ stands for quantities uniformly bounded in $a$ and $\lambda$. For $a \in \partial M, \epsilon$ and $\delta$ positive and small and $\lambda$ large, $O_{a, \epsilon, \delta}(1)$ and $O_{a, \lambda}(1)$ stand, respectively, for quantities which are bounded uniformly in $a, \delta$ and $\epsilon$ and in $a$ and $\lambda$. For $a \in \partial M, \epsilon$ positive and small and $\lambda$ large, $o_{a, \epsilon}(1)$ and $o_{a, \lambda}(1)$ stand, respectively, for quantities which tend to 0 uniformly in $a$ as $\epsilon$ tends to 0 and as $\lambda$ tends to $+\infty$. For $A \in(\partial M)^{2}$ and $\lambda$ large, $O_{A, \lambda}(1)$ and $o_{A, \lambda}(1)$ stand, respectively, for quantities which are bounded uniformly in $A$ and $\lambda$, and which tend to 0 uniformly in $A$ as $\lambda$ tends to $+\infty$. For $p \in \mathbb{N}^{*}, A \in(\partial M)^{p}, \bar{\alpha} \in \Delta_{p-1}$ and $\lambda$ large, $O_{A, \bar{\alpha}, \lambda}(1)$ and $o_{A, \bar{\alpha}, \lambda}(1)$ stand, respectively, for quantities which are uniformly bounded in $p, A, \bar{\alpha}$ and $\lambda$ and for quantities which tend to 0 uniformly in $p, A$ and $\bar{\alpha}$ as $\lambda$ tends to $+\infty$. For $x \in \mathbb{R}$, we will use the notation $O(x)$ and $o(x)$ to mean, respectively, $|x| O(1)$ and $|x| o(1)$ where $O(1)$ and $o(1)$ will be specified in all the contexts where they are used. Large positive constants are usually denoted by $C$ and the value of $C$ is allowed to vary from formula to formula and also within the same line. Similarly small positive constants are denoted by $c$ and their values may vary from formula to formula and also within the same line. The symbol $\sum_{i \neq j}$ always means a double sum over the associated index set under the assumption $i \neq j$.

For $X$ a topological space, $H_{*}(X)$ will denote the singular homology of $X$ with $\mathbb{Z}_{2}$ coefficients and $H^{*}(X)$ for the cohomology. For $Y$ a subspace of $X, H_{*}(X, Y)$ will stand for the relative homology. The symbol $\frown$ will denote the cap product between cohomology and homology. For a map $f: X \rightarrow Y$, with $X$ and $Y$ topological spaces, $f_{*}$ stands for the induced map in homology and $f^{*}$ for the induced map in cohomology. For $p \in \mathbb{N}$, we set

$$
W_{p}:=\left\{u \in W_{+}^{1,2}(\bar{M}): \quad \mathcal{E}_{g}(u) \leq(p+1)^{\frac{1}{n-1}} \mathcal{Q}\left(B^{n}\right)\right\}
$$

where

$$
\mathcal{Q}\left(B^{n}\right):=\mathcal{Q}\left(B^{n}, \partial B^{n}, g_{B^{n}}\right)
$$

For a Riemannian metric $\bar{g}$ defined on $\bar{M}$, we denote by $G_{\bar{g}}$ the Green's function of $\left(L_{\bar{g}}, B_{\bar{g}}\right)$ satisfying the normalization

$$
\lim _{\overline{\bar{g}_{\bar{g}}}(a, x) \longrightarrow 0}\left(\bar{d}_{\bar{g}}(a, x)\right)^{n-2} G_{\bar{g}}(a, x)=1,
$$


and set

$$
G:=G_{g}
$$

Using the existence of conformal normal Fermi coordinates (see [55]) and recalling that $\partial M$ is totally geodesic in $(\bar{M}, g)$, we have that for every large positive integer $m$ and for every $a \in \partial M$, there exists a positive function $u_{a} \in C^{\infty}(\bar{M})$ such that the metric

$$
g_{a}:=u_{a}^{\frac{4}{n-2}} g
$$

verifies

$$
\operatorname{det}_{a}(x)=1+O_{a, x}\left(\left(\bar{d}_{g_{a}}(x, a)\right)^{m}\right) \quad \text { for } \quad x \in B_{a}^{g_{a}}\left(\varrho_{a}\right),
$$

with $O_{a, x}(1)$ meaning bounded by a constant independent of $a$ and $x, \quad 0<\varrho_{a}<\min \left\{\frac{i n j_{g_{a}}(\bar{M})}{10}, \frac{i n j_{\hat{g}_{a}}(\partial M)}{10}\right\}$. Moreover, we can take the family of functions $u_{a}, g_{a}$ and $\varrho_{a}$ such that

$$
\text { the maps } a \longrightarrow u_{a}, g_{a} \text { are } C^{0} \text { and } \frac{1}{4} \geq \varrho_{a} \geq \varrho_{0}>0
$$

for some small positive $\varrho_{0}$ satisfying $\varrho_{0}<\min \left\{\frac{i n j_{g}(\bar{M})}{10}, \frac{i n j_{\hat{g}}(\partial M)}{10}\right\}$, and

$$
\begin{aligned}
& \left\|u_{a}\right\|_{C^{2}(\bar{M})}=O_{a}(1), \frac{1}{\bar{C}^{2}} g \leq g_{a} \leq \bar{C}^{2} g, \quad a \in \bar{M}, \\
& u_{a}(a)=1 \text { and } H_{a}:=H_{g_{a}}=0, \\
& u_{a}(x)=1+O_{a, x}\left(\bar{d}_{g_{a}}^{2}(a, x)\right)=1+O_{a, x}\left(\bar{d}_{g}^{2}(a, x)\right),
\end{aligned}
$$

for $x \in B_{a}^{g_{a}}\left(\varrho_{0}\right) \supset B_{a}\left(\frac{\varrho_{0}}{2 \bar{C}}\right)$ and some large positive constant $\bar{C}$ independent of $a$ and $O_{a, x}(1)$ is as in (10). We remark that in general, namely for $g$ arbitrary, we know from [55] that

$$
H_{a}=O_{a, x}\left(d_{\hat{g}_{a}}^{m-1}(a, x)\right)=1+O_{a, x}\left(d_{\hat{g}}^{m-1}(a, x)\right),
$$

for $x \in \hat{B}_{a}^{g_{a}}\left(\varrho_{0}\right) \supset \hat{B}_{a}\left(\frac{\varrho_{0}}{2 \bar{C}}\right)$, where $\hat{g}_{a}:=\left.g_{a}\right|_{\partial M}$, and $O_{a, x}$ is as in (11). We point out that the latter suffices for our purpose, since we will be working with the Almaraz $[\mathbf{3}]-$ Chen $[\mathbf{3 4}]$ 's bubbles. However, we quickly provide some indications on how one can use Marques[55]'s work to achieve $H_{a}=0$ in the totally geodesic case (even under the only assumption $H_{g}=0$ on $\left.\partial M\right)$. From Marques[55], one has a function $\tilde{u}_{a}>0$ and smooth such that $\tilde{g}_{a}:=\tilde{u}_{a}^{\frac{4}{n-2}} g$ verifies (10)(11) with some $\tilde{C}$ and some $\tilde{\varrho}_{a}>0$ except the global vanishing of the associated mean curvature, but one always has its local vanishing, namely $\frac{\partial \tilde{u}_{a}}{\partial n_{g}}=0$ in $\hat{B}_{a}^{\tilde{g}_{a}}\left(\tilde{\varrho}_{a}\right)$ (realling that we are in the totally geodesic case), see (1) of Proposition 3.2 in [55], however, as remark in the proof of Proposition in [55], the local vanishing of the mean curvature always holds, namely for $g$ arbitrary. Thus, setting $u_{a}:=\tilde{\chi}_{a} u_{a}+\left(1-\tilde{\chi}_{a}\right)$ with $\tilde{\chi}_{a}=\chi\left(\frac{\tilde{C} \bar{d}_{g}(a, \cdot)}{\tilde{\varrho}_{a}}\right)$, for some large $\bar{C}$ such that $g_{a}:=u_{a}^{\frac{4}{n-2}} g$ verifies 
the comparability $\frac{1}{\bar{C}^{2}} g \leq g_{a} \leq \bar{C}^{2}$, and $\chi$ is a cut-off function defined on $\overline{\mathbb{R}}_{+}$satisfying $\chi$ is non-negative, $\chi(t)=1$ if $t \leq \frac{1}{2}$ and $\chi(t)=0$ if $t \geq 1$, then one has that $g_{a}=\tilde{g}_{a}$ in $B_{a}^{g_{a}}\left(\varrho_{a}\right)$ with $\varrho_{a}:=\frac{\tilde{\varrho}_{a}}{2 \bar{C} \tilde{C}}$ and $\frac{\partial u_{a}}{\partial n_{g}}=0$ on $\partial M$. Hence, recalling again that we are in the totally geodesic case (however, just $H_{g}=0$ on $\partial M$ suffices), one has that the mean curvature $H_{a}$ of $g_{a}$ vanishes identically on $\partial M$, thus clearly $g_{a}$ satisfies fully (10)-(11) as desired. Now, having finished to give indications on how one can achieve $H_{a}=0$ on $\partial M$, we continue to make some definitions. For $a \in \partial M$ and $\epsilon$ positive, we recall that the standard bubbles of the geometric problem under study are defined as follows:

$$
\delta_{a, \epsilon}(x):=\left(\frac{\epsilon}{\left(\epsilon+x_{n}\right)^{2}+\left|x^{\prime}\right|^{2}}\right)^{\frac{n-2}{2}}, \quad x \in B_{a}^{g_{a}}\left(\varrho_{0}\right)
$$

where $\left(x^{\prime}, x_{n}\right)$ is the Fermi normal coordinate of $x$ with respect to $g_{a}$ at $a$. For $a \in \partial M$ and $0<r<\varrho_{0}$, we set also

$$
\begin{aligned}
& G_{a}:=G_{g_{a}}, \quad \exp _{a}^{a}=\exp _{a}^{g_{a}}, \quad \exp _{a}^{a}=\exp _{a}^{\hat{g}_{a}}, \\
& B_{a}^{a}(r):=B_{a}^{g_{a}}(r) \quad \text { and } \quad \hat{B}_{a}^{a}(r):=\hat{B}_{a}^{g_{a}}(r) .
\end{aligned}
$$

On the other hand, the conformal invariance properties of the couple conformal Laplacian and conformal Neumann operator imply

$$
\begin{aligned}
& \mathcal{E}_{g}(u)=\mathcal{E}_{g_{a}}\left(u_{a}^{-1} u\right), \quad \oint_{\partial M} u^{\frac{2(n-1)}{n-2}} d S_{g}=\oint_{\partial M}\left(u_{a}^{-1} u\right)^{\frac{2(n-1)}{n-2}} d S_{g_{a}}, \\
& G_{g}(x, y)=G_{a}(x, y) u_{a}(x) u_{a}(y)
\end{aligned}
$$

for $(x, y) \in \bar{M}^{2}, a \in \partial M$ and $u \in W_{+}^{1,2}(\bar{M})$. We also define

$$
\begin{aligned}
& c_{0}:=(n-2), \quad c_{1}:=\int_{\mathbb{R}^{n-1}}\left(\frac{1}{1+|x|^{2}}\right)^{n-1} d x, \text { and } \\
& c_{2}:=4 \frac{n-1}{n-2} \int_{\mathbb{R}_{+}^{n}}\left|\nabla\left[\left(\frac{1}{\left(1+x_{n}\right)^{2}+\left|x^{\prime}\right|^{2}}\right)^{\frac{n-2}{2}}\right]\right|^{2} d x .
\end{aligned}
$$

Furthermore, we set

$$
c_{3}:=\int_{\mathbb{R}^{n-1}}\left(\frac{1}{1+|x|^{2}}\right)^{\frac{n}{2}} d x
$$

and define the following quantity which depends only on $(\bar{M}, g)$

$$
c_{g}=\frac{c_{3}}{4 c_{1}} \min _{\left((\partial M)^{2}\right)^{*}} G,
$$

and see above for the definition of $\left((\partial M)^{2}\right)^{*}, G, \quad c_{1}$ and $c_{3}$. We recall that the numbers $c_{i}(i=0,1,2)$ and $\mathcal{Q}\left(B^{n}\right)$ verify the following 
relation:

$$
c_{2}=c_{0} c_{1} \quad \text { and } \quad \mathcal{Q}\left(B^{n}\right)=\frac{c_{2}}{c_{1}^{\frac{n-2}{n-1}}} .
$$

Moreover, for $p \in \mathbb{N}^{*}, A:=\left(a_{1}, \cdots, a_{p}\right) \in(\partial M)^{p}, \bar{\lambda}:=\left(\lambda_{1}, \cdots, \lambda_{p}\right) \in$ $\left(\mathbb{R}_{+}\right)^{p}$, we associate the following quantities (which appear in the analysis of diverging PS sequences of the Euler-Lagrange functional $\mathcal{E}_{g}$ )

$$
\varepsilon_{i, j}:=\varepsilon_{i, j}(A, \bar{\lambda}):=\frac{c_{3}}{\left(\frac{\lambda_{i}}{\lambda_{j}}+\frac{\lambda_{j}}{\lambda_{i}}+\lambda_{i} \lambda_{j} G^{\frac{2}{2-n}}\left(a_{i}, a_{i}\right)\right)^{\frac{n-2}{2}}},
$$

where $i, j=1, \cdots, p, \quad i \neq j$.

Now, we are going to present some topological properties of the space of formal barycenter of $\partial M$ that we will need for our algebraic topological argument for existence. To do that, for $p \in \mathbb{N}^{*}$, we recall that the set of formal barycenters of $\partial M$ of order $p$ is defined as follows:

$$
B_{p}(\partial M):=\left\{\sum_{i=1}^{p} \alpha_{i} \delta_{a_{i}}: a_{i} \in \partial M, \alpha_{i} \geq 0, \sum_{i=1}^{p} \alpha_{i}=1\right\}
$$

and set

$$
B_{0}(\partial M)=\emptyset .
$$

Furthermore, we have the existence of $\mathbb{Z}_{2}$ orientation classes $w_{p} \in$ $H_{n p-1}\left(B_{p}(\partial M), B_{p-1}(\partial M)\right)$ and that the cap product acts as follows (see, for example, [14], page 263, line 5):

$$
\begin{aligned}
H^{l}\left((\partial M)^{p} / \sigma_{p}\right) & \times H_{k}\left(B_{p}(\partial M), B_{p-1}(\partial M)\right) \\
& \frown H_{k-l}\left(B_{p}(\partial M), B_{p-1}(\partial M)\right) .
\end{aligned}
$$

On the other hand, since $\partial M$ is a closed $(n-1)$-dimensional manifold, then we have

$$
\text { an orientation class } 0 \neq O_{\partial M}^{*} \in H^{n-1}(\partial M) \text {. }
$$

Moreover, there is a natural way to see (precisely to transfer) $O_{\partial M}^{*} \in$ $H^{n-1}(\partial M)$ as a nontrivial element of $H^{n-1}\left((\partial M)^{p} / \sigma_{p}\right)$ (see [12] formula (6.159) or [14] page 265 and [45] page 161 and Definition 23), namely

$$
O_{\partial M}^{*} \simeq O_{p}^{*} \text { with } 0 \neq O_{p}^{*} \in H^{n-1}\left((\partial M)^{p} / \sigma_{p}\right) .
$$

We recall briefly the construction of $O_{p}^{*}$, and for more details see [12] or [14]. The main ingredient in the definition of $O_{p}^{*}$ is a transfer map (see $[\mathbf{1 7}]$ )

$$
\operatorname{tr}: H^{n-1}\left((\partial M)^{p} / \sigma_{1} \times \sigma_{p-1}\right) \longrightarrow H^{n-1}\left((\partial M)^{p} / \sigma_{p}\right)
$$

where $\sigma_{1} \times \sigma_{p-1}$ is the subgroup of $\sigma_{p}$ consisting of elements of $\sigma_{p}$ which map 1 to 1 . We now describe the main map and facts that are 
used in the construction of the map $t r$, and for more details see [12] or [45]. The first fact is the following equivalence:

$$
(\partial M)^{p} / \sigma_{p} \simeq\left((\partial M)^{p} / \sigma_{1} \times \sigma_{p-1}\right) /\left(\sigma_{p} / \sigma_{1} \times \sigma_{p-1}\right),
$$

and the associated projection

$$
q:(\partial M)^{p} / \sigma_{1} \times \sigma_{p-1} \longrightarrow(\partial M)^{p} / \sigma_{p}
$$

The next fact and the main map used in the construction of $t r$ is Poincaré duality and $q_{*}$. With the transfer map $t r$ at hand, the next ingredient in the definition of $O_{p}^{*}$ is the projection

$$
\pi:(\partial M)^{p} / \sigma_{1} \times \sigma_{p-1} \longrightarrow \partial M .
$$

Indeed, (21), (23) and (24) defines $O_{p}^{*}$ via the following formula:

$$
O_{p}^{*}:=\operatorname{tr} \circ \pi^{*}\left(O_{\partial M}^{*}\right) \text {. }
$$

Now, identifying $O_{\partial M}^{*}$ and $O_{p}^{*}$ via (22) and using (20) we have the following well-know formula, see $[\mathbf{1 2}]$ formula (25), or [14] and [50] for related issues.

Lemma 2.1. There holds

$$
\begin{aligned}
& H^{n-1}\left((\partial M)^{p} / \sigma_{p}\right) \times H_{n p-1}\left(B_{p}(\partial M), B_{p-1}(\partial M)\right) \\
& \leftrightharpoons H_{n p-n}\left(B_{p}(\partial M), B_{p-1}(\partial M)\right) \\
& \stackrel{\partial}{\longrightarrow} H_{n p-n-1}\left(B_{p-1}(\partial M), B_{p-2}(\partial M)\right),
\end{aligned}
$$

and

$$
\omega_{p-1}=\partial\left(O_{\partial M}^{*} \frown w_{p}\right) .
$$

Next, we are going to discuss some important properties of the Almaraz[3]-Chen[34]'s bubbles. Using the techniques of Brendle[21], Almaraz[3]-Chen[34] have introduced a family of bubbles which verify the same properties as the Brendle[21]'s bubbles and the BrendleChen[23]'s bubbles. Indeed, for $\delta$ small, they define a family of bubbles $v_{a, \epsilon, \delta}$ (see page 2643 in [3] and page 16 in [34]), $a \in \partial M$ and $\epsilon$ positive and small such that they can replace the standard bubbles in the analysis of diverging PS sequences of $\mathcal{E}_{g}$ and more importantly verify a sharp energy estimate. Precisely, $v_{a, \epsilon, \delta}$ is defined as a suitable perturbation of the standard bubbles glued with an appropriate scale of the Green's function $G_{a}$ centered at $a$ as follows:

$$
v_{a, \epsilon, \delta}(\cdot)=\chi_{\delta}(\cdot)\left(\delta_{a, \epsilon}(\cdot)+w_{a, \epsilon}(\cdot)\right)+\left(1-\chi_{\delta}(\cdot)\right) \epsilon^{\frac{n-2}{2}} G_{a}(a, \cdot),
$$

where

$$
\chi_{\delta}(x):=\chi\left(\frac{\bar{d}_{g_{a}}(a, x)}{\delta}\right),
$$

and $\chi$ is a cut-off function defined on $\overline{\mathbb{R}}_{+}$satisfying $\chi$ is non-negative, $\chi(t)=1$ if $t \leq 1$ and $\chi(t)=0$ if $t \geq 2, \delta_{a, \epsilon}$ is defined as in (12), 
$G_{a}(a, \cdot)$ is defined as in (13), and in Fermi normal coordinates around $a$ with respect to $g_{a}$ we have that $w_{a, \epsilon}$ satisfies the pointwise estimate

$$
\left|\partial^{\beta} w_{a, \epsilon}(x)\right| \leq C_{n}(|\beta|) \frac{\epsilon^{\frac{n-2}{2}}}{\left(\epsilon^{2}+r^{2}\right)^{\frac{n-4+\beta}{2}}},
$$

where $r=\bar{d}_{g_{a}}(a, x), x \in B_{a}^{a}\left(\varrho_{0}\right), \varrho_{0}$ is as in (11) and $C_{n}(|\beta|)$ is a large positive constant which depends only on $n$ and $|\beta|$. Furthermore, recalling the assumption $\mathcal{Z}_{g}=\partial M$, we have that $v_{a, \epsilon, \delta}$ verifies the following energy estimate which is a weak form (but sufficient for the purpose of this paper) of a combination either of Proposition 3.11 in [3] and Proposition 3.9 in [3] or of Proposition 3.11 in [3] and Proposition 9 in [34] (for a reader who feels comfortable with the property $H_{a}=0$ ).

Lemma 2.2. There exists $0<\delta_{0} \leq \varrho_{0}$ small such that for every $0<2 \epsilon \leq \delta \leq \delta_{0}$ and for every $a \in \partial M$, there holds

$$
\begin{aligned}
\left\langle L_{g_{a}} v_{a, \epsilon, \delta}, v_{a, \epsilon, \delta}\right\rangle & +\left\langle B_{g_{a}} v_{a, \epsilon, \delta}, v_{a, \epsilon, \delta}\right\rangle \\
& \leq \mathcal{Q}\left(B^{n}\right)\left(\oint_{\partial M} v_{a, \epsilon, \delta}^{\frac{2(n-1)}{n-2}} d S_{g_{a}}\right)^{\frac{n-2}{n-1}}-\epsilon^{n-2} \mathcal{I}(a, \delta) \\
& +O_{a, \epsilon, \delta}\left(\delta \epsilon^{n-2}+\epsilon^{n-1} \delta^{-n+1}\right),
\end{aligned}
$$

where $\mathcal{Q}\left(B^{n}\right)$ is defined by $(8), \mathcal{I}(a, \delta)$ is a flux integral verifying $\mathcal{I}(a, \delta)=\mathcal{I}(a)+o_{a, \delta}(1), \quad \mathcal{I}(a)=O_{a}(1)$, and for the meaning of $o_{a, \delta}(1)$ $O_{a}(1)$ and $O_{a, \epsilon, \delta}(1)$, see Section 2.

On the other hand, using the work of Almaraz[3] (Lemma 3.15 in $[3])$, we have that the $v_{a, \epsilon, \delta}$ 's verify the following interaction estimates.

Lemma 2.3. There exists a large constant $C_{1}>0$ such that for every $2 \epsilon_{1} \leq 2 \epsilon_{2} \leq \delta^{2} \leq \delta_{0}$ and every $a_{1}, a_{2} \in \partial M$, there holds

$$
\begin{gathered}
\int_{M} v_{a_{1}, \epsilon_{1}, \delta}\left|L_{g_{a_{2}}} v_{a_{2}, \epsilon_{2}, \delta}\right| d V_{g_{a_{2}}} \\
+\oint_{\partial M} v_{a_{1}, \epsilon_{1}, \delta}\left|B_{g_{a_{2}}} v_{a_{2}, \epsilon_{2}, \delta}-c_{0} v_{a_{2}, \epsilon_{2}, \delta}^{\frac{n}{n-2}}\right| d S_{g_{a_{2}}} \\
\leq C_{1}\left(\delta+\frac{\epsilon_{2}}{\delta}\right)\left(\frac{\epsilon_{2}^{2}+d_{g_{a_{2}}}^{2}\left(a_{1}, a_{2}\right)}{\epsilon_{1} \epsilon_{2}}\right)^{\frac{2-n}{2}},
\end{gathered}
$$

where $c_{0}$ is defined by (15).

Furthermore, using (25), it is easy to see that the following estimate holds:

Lemma 2.4. Assuming that $0<\epsilon \leq \delta_{0}^{n}$ and $a \in \partial M$, then we have

$$
\oint_{\partial M} v_{a, \epsilon, \epsilon}^{\frac{2(n-1)}{n-2}} d S_{g_{a}}=c_{1}+o_{a, \epsilon}(1),
$$

where $c_{1}$ is as in (15), and for the meaning of $o_{a, \epsilon}(1)$, see Section 2. 
Thus, setting

$$
v_{a}^{\lambda}:=v_{a, \frac{1}{\lambda},\left(\frac{1}{\lambda}\right)^{\frac{1}{n}}}, \quad a \in \partial M, \quad \lambda \geq \frac{1}{\delta_{0}^{n}},
$$

and

$$
\varphi_{a, \lambda}:=u_{a} v_{a}^{\lambda}, \quad a \in \partial M, \quad \lambda \geq \frac{1}{\delta_{0}^{n}},
$$

where $u_{a}$ is as in (10) and $\delta_{0}$ is still given by Lemma 2.2, we have clearly that Lemma 2.2, Lemma 2.3 and Lemma 2.4 combined with (14) imply the following lemmata which will play an important role in our application of the barycenter technique of Bahri-Coron[14]:

Lemma 2.5. Assuming that $a \in \partial M$ and $\lambda \geq \frac{1}{\delta_{0}^{n}}$, then the following estimate holds:

$$
\mathcal{E}_{g}\left(\varphi_{a, \lambda}\right) \leq \mathcal{Q}\left(B^{n}\right)\left(1+O_{a, \lambda}\left(\frac{1}{\lambda^{n-2}}\right)\right),
$$

where $\mathcal{Q}\left(B^{n}\right)$ is as in (8) and for the meaning of $O_{a, \lambda}(1)$, see Section 2.

Lemma 2.6. There exists a large constant $C_{2}>0$ such that for every $a_{1}, a_{2} \in \partial M$ and for every $\lambda \geq \frac{1}{\delta_{0}^{n}}$, we have

$$
\begin{aligned}
& \int_{M} \varphi_{a_{1}, \lambda}\left|L_{g_{a_{2}}} \varphi_{a_{2}, \lambda}\right| d V_{g_{a_{2}}}+\oint_{\partial M} \varphi_{a_{1}, \lambda}\left|B_{g_{a_{2}}} \varphi_{a_{2}, \lambda}-c_{0} \varphi_{a_{2}, \lambda}^{\frac{n}{n-2}}\right| d S_{g_{a_{2}}} \\
& \leq C_{1}\left(\frac{1}{\lambda}\right)^{\frac{1}{n}}\left(1+\lambda^{2} d_{g_{a_{2}}}^{2}\left(a_{1}, a_{2}\right)\right)^{\frac{2-n}{2}}
\end{aligned}
$$

where $c_{0}$ is as in (15).

Lemma 2.7. Assuming that $a \in \partial M$ and $\lambda \geq \frac{1}{\delta_{0}^{n}}$, then there holds

$$
\oint_{\partial M} \varphi_{a, \lambda}^{\frac{2(n-1)}{n-2}} d S_{g_{a}}=c_{1}+o_{a, \lambda}(1)
$$

where $c_{1}$ is as in (15) and for the meaning of $o_{a, \lambda}(1)$, see Section 2.

On the other hand, using (25)-(26) and (27), we have that $v_{a}^{\lambda}$ decomposes as follows:

$$
v_{a}^{\lambda}(\cdot)=\chi^{\lambda}(\cdot)\left(\delta_{a}^{\lambda}(\cdot)+w_{a}^{\lambda}(\cdot)\right)+\left(1-\chi^{\lambda}(\cdot)\right) \frac{G_{a}(a, \cdot)}{\lambda^{\frac{n-2}{2}}},
$$

where

$$
w_{a}^{\lambda}:=w_{a, \frac{1}{\lambda}}, \quad \delta_{a}^{\lambda}:=\delta_{a, \frac{1}{\lambda}}, \quad \text { and } \quad \chi^{\lambda}=\chi_{\left(\frac{1}{\lambda}\right) \frac{1}{n}},
$$

and $w_{a}^{\lambda}$ satisfies the following pointwise estimate:

$$
\left|\partial^{\beta} w_{a}^{\lambda}(x)\right| \leq C_{n}(|\beta|) \frac{\lambda^{\frac{n-6}{2}+\beta}}{\left(1+\lambda^{2} r^{2}\right)^{\frac{n-4+\beta}{2}}},
$$


where $r=\bar{d}_{g_{a}}(a, x)$ and $x \in B_{a}^{a}\left(\varrho_{0}\right)$. Now, for $p \in \mathbb{N}^{*}$, and $A:=$ $\left(a_{1}, \cdots, a_{p}\right) \in(\partial M)^{p}$ and $\lambda \geq \frac{1}{\delta_{0}^{n}}$, we associate the following quantities:

$$
\epsilon_{i, j}:=\epsilon_{i, j}(A, \lambda):=\oint_{\partial M} \varphi_{a_{i}, \lambda}^{\frac{n}{n-2}} \varphi_{a_{j}, \lambda} d S_{g}, \quad i, j=1, \cdots, p, \quad i \neq j .
$$

Using (28), (29)-(32), we have the following lemma which provides self- and interaction estimates and a relation between $\epsilon_{i, j}(A, \lambda)$ and $\varepsilon_{i, j}(A, \bar{\lambda})$ with $\bar{\lambda}:=(\lambda, \cdots, \lambda)$; for the meaning of $\varepsilon_{i, j}(A, \bar{\lambda})$ see $(18)$.

Lemma 2.8. Assuming that $p \in \mathbb{N}^{*}, A:=\left(a_{1}, \cdots, a_{p}\right) \in(\partial M)^{p}$ and $\lambda \geq \frac{1}{\delta_{0}^{n}}$, then

1) For every $i, j=1, \cdots, p$ with $i \neq j$, we have i)

$$
\epsilon_{i, j} \longrightarrow 0 \Longleftrightarrow \varepsilon_{i, j} \longrightarrow 0,
$$

where $\varepsilon_{i, j}:=\varepsilon_{i, j}(A, \bar{\lambda})$ with $\bar{\lambda}:=(\lambda, \cdots, \lambda)$ and $\epsilon_{i, j}:=\epsilon_{i, j}(A, \lambda)$, and for their definitions see, respectively, (18) and (32).

ii) There exists $0<C_{3}<\infty$ independent of $p, A$ and $\lambda$ such that the following estimate holds:

$$
C_{3}^{-1}<\frac{\epsilon_{i, j}}{\varepsilon_{i, j}}<C_{3}
$$

iii) If $\varepsilon_{i, j} \longrightarrow 0$, then

$$
\epsilon_{i, j}=\left(1+o_{\varepsilon_{i, j}}(1)\right) \varepsilon_{i, j},
$$

and for the meaning of $o_{\varepsilon_{i, j}}(1)$, see Section 2 .

2) For every $i=1, \cdots, p$, there holds

$$
\left\langle L_{g} \varphi_{a_{i}, \lambda}, \varphi_{a_{i}, \lambda}\right\rangle+\left\langle B_{g} \varphi_{a_{i}, \lambda}, \varphi_{a_{i}, \lambda}\right\rangle=c_{0}\left(1+o_{a_{i}, \lambda}(1)\right) \oint_{\partial M} \varphi_{a_{i}, \lambda}^{\frac{2(n-1)}{n-2}} d S_{g},
$$

where $c_{0}$ is given by (15) and for the meaning of $o_{a_{i}, \lambda}(1)$, see Section 2. 3) For every $i, j=1, \cdots, p$ with $i \neq j$, there holds

$$
\begin{aligned}
\left\langle L_{g} \varphi_{a_{i}}, \varphi_{a_{j}}\right\rangle+\left\langle B_{g} \varphi_{a_{i}, \lambda}, \varphi_{a_{j}, \lambda}\right\rangle & =\left(1+o_{A_{i, j}, \lambda}(1)\right) c_{0} \epsilon_{i, j}, \quad \text { and } \\
\epsilon_{j, i} & =\left(1+o_{A_{i, j}, \lambda}(1)\right) \epsilon_{i, j},
\end{aligned}
$$

where $A_{i, j}:=\left(a_{i}, a_{j}\right)$ and for the meaning of $o_{A_{i, j}, \lambda}(1)$, see Section 2.

Proof. To prove Lemma 2.8, we use the same strategy as in [57]. First of all, to simplify notation, for every $i=1, \cdots, p$, we set

$$
\begin{aligned}
& \varphi_{i}:=\varphi_{a_{i}, \lambda}, \quad v_{i}:=v_{a_{i}}^{\lambda}, \quad \delta_{i}:=\delta_{a_{i}}^{\lambda}, \quad w_{i}:=w_{a_{i}}^{\lambda}, G_{i}(\cdot):=G_{a_{i}}\left(a_{i}, \cdot\right), \\
& B_{i}:=\hat{B}_{a_{i}}^{a_{i}}\left(\delta_{0}\right), \quad \exp _{i}:=\exp _{a_{i}}^{a_{i}} \text { and } u_{i}:=u_{a_{i}},
\end{aligned}
$$

and for the meaning of $\hat{B}_{a_{i}}^{a_{i}}\left(\delta_{0}\right)$ and $\hat{\operatorname{xxp}}_{a_{i}}^{a_{i}}$, see Section 2. Next, using (31) and (33), we have that $v_{i}$ verifies the following pointwise estimate:

$$
v_{i}=\left(1+o_{a_{i}, \lambda}(1)\right)\left(\chi^{\lambda} \delta_{i}+\left(1-\chi^{\lambda}\right) \frac{G_{i}}{\lambda^{\frac{n-2}{2}}}\right) .
$$


Moreover, using (9), (12), (30) and (33), we obtain (on $\partial M$ )

$$
\begin{aligned}
\chi^{\lambda} \delta_{i} & =\chi^{\lambda}\left(\frac{\lambda}{1+\lambda^{2} r^{2}}\right)^{\frac{n-2}{2}}=\chi^{\lambda}\left(\frac{\lambda}{1+\lambda^{2} G_{i}^{\frac{2}{2-n}} \frac{r^{2}}{G_{i}^{\frac{2}{2-n}}}}\right)^{\frac{n-2}{2}} \\
& =\left(1+o_{a_{i}, \lambda}(1)\right) \chi^{\lambda}\left(\frac{\lambda}{1+\lambda^{2} G_{i}^{\frac{2}{2-n}}}\right)^{\frac{n-2}{2}},
\end{aligned}
$$

where $r$ is as in (26) with $a$ replaced by $a_{i}$, and

$$
\begin{aligned}
\left(1-\chi^{\lambda}\right)\left(\frac{\lambda}{1+\lambda^{2} G_{i}^{\frac{2}{2-n}}}\right)^{\frac{n-2}{2}} & =\left(1-\chi^{\lambda}\right) \frac{G_{i}}{\lambda^{\frac{n-2}{2}}}\left(\frac{1}{1+O_{a_{i}, \lambda}\left(\left|\frac{1}{\lambda}\right| \frac{2(n-1)}{n}\right)}\right) \\
& =\left(1+o_{a_{i}, \lambda}(1)\right)\left(1-\chi^{\lambda}\right) \frac{G_{i}}{\lambda^{\frac{n-2}{2}}} .
\end{aligned}
$$

Hence, combining (34) and (35), we obtain

$$
v_{i}=\left(1+o_{a_{i}, \lambda}(1)\right)\left(\frac{\lambda}{1+\lambda^{2} G_{i}^{\frac{2}{2-n}}}\right)^{\frac{n-2}{2}} .
$$

Now, using (11), (28), (32), (33) and (36), we derive the following estimate for $\epsilon_{i, j}(i, j=1, \cdots, p$ and $i \neq j)$ :

$$
\begin{aligned}
\epsilon_{i, j}= & \oint_{\partial M} v_{i}^{\frac{n}{n-2}} v_{j} \frac{u_{j}}{u_{i}} d S_{g_{a_{i}}}=\int_{B_{i}} v_{i}^{\frac{n}{n-2}} v_{j} \frac{u_{j}}{u_{i}} d S_{g_{a_{i}}}+O_{A_{i, j}, \lambda}\left(\lambda^{1-n}\right) \\
= & \left(1+o_{A_{i, j}, \lambda}(1)\right) u_{j}\left(a_{i}\right) \\
& \quad \int_{B^{\lambda}(0)}\left(\frac{1}{1+|x|^{2}}\right)^{\frac{n}{2}}\left[\frac{1}{1+\lambda^{2} G_{j}^{\frac{2}{2-n}}\left(\exp _{i}\left(\frac{x}{\lambda}\right)\right)}\right]^{\frac{n-2}{2}} d x \\
& +O_{A_{i, j}, \lambda}\left(\lambda^{1-n}\right),
\end{aligned}
$$

where

$$
B^{\lambda}(0):=B_{0}^{\mathbb{R}^{n-1}}\left(\lambda \delta_{0}\right)
$$

and for the meaning of $B_{0}^{\mathbb{R}^{n-1}}\left(\lambda \delta_{0}\right)$, see Section 2. From (37) it follows that:

$$
\epsilon_{i, j} \longrightarrow 0 \Longleftrightarrow \lambda^{2} G_{j}^{\frac{2}{2-n}}\left(a_{i}\right) \longrightarrow+\infty \Longleftrightarrow \varepsilon_{i, j} \longrightarrow 0 .
$$

Thus, we have that the proof of i) of point 1) is complete. Now, since $\epsilon_{i, j}$ and $\varepsilon_{i, j}$ are bounded by definition, then thanks to (38), to prove 
ii) of point 1), we can assume without loss of generality that

$$
\lambda^{2} G_{j}^{\frac{2}{2-n}}\left(a_{j}\right) \gg 1 .
$$

Thus under the latter assumption, setting

$$
\mathcal{A}=B_{0}^{\mathbb{R}^{n-1}}\left(\gamma \lambda \sqrt{G_{j}^{\frac{2}{2-n}}\left(a_{i}\right)}\right),
$$

for $\gamma>0$ small and using Taylor expansion, we obtain that the following estimate holds on $\mathcal{A}$ :

$$
\begin{aligned}
& \left(\frac{1}{1+\lambda^{2} G_{j}^{\frac{2}{2-n}}\left(\exp _{i}\left(\frac{x}{\lambda}\right)\right)}\right)^{\frac{n-2}{2}} \\
& =\left(\frac{1}{1+\lambda^{2} G_{j}^{\frac{2}{2-n}}\left(a_{i}\right)}\right)^{\frac{n-2}{2}}\left(\frac{1}{1+\frac{\lambda^{2}\left[G_{j}^{2-n}\left(\exp _{i}\left(\frac{x}{\lambda}\right)\right)-G_{j}^{2-n}\left(a_{i}\right)\right]}{1+\lambda^{2} G_{j}^{2-n}}\left(a_{i}\right)}\right)^{\frac{n-2}{2}} \\
& =\left(\frac{1}{1+\lambda^{2} G_{j}^{\frac{2}{2-n}}\left(a_{i}\right)}\right)^{\frac{n-2}{2}}\left(\frac{1}{1+\frac{\lambda \nabla G_{j}^{\frac{2}{2-n}}\left(a_{i}\right) x+O\left(|x|^{2}\right)}{1+\lambda^{2} G_{j}^{\frac{2}{2-n}}\left(a_{i}\right)}}\right)^{\frac{n-2}{2}} \\
& =\left(\frac{1}{1+\lambda^{2} G_{j}^{\frac{2}{2-n}}\left(a_{i}\right)}\right)^{\frac{n-2}{2}}-\frac{n-2}{2} \lambda\left(\frac{1}{1+\lambda^{2} G_{j}^{\frac{2}{2-n}}\left(a_{i}\right)}\right)^{\frac{n}{2}} \nabla G_{j}^{\frac{2}{2-n}}\left(a_{i}\right) x \\
& +O\left(\frac{|x|^{2}}{\left(1+\lambda^{2} G_{j}^{\frac{2}{2-n}}\left(a_{i}\right)\right)^{\frac{n}{2}}}\right) .
\end{aligned}
$$

Now, combining (37) and (40), we obtain

$$
\begin{aligned}
\epsilon_{i, j}= & \left(1+o_{A_{i, j}, \lambda}(1)\right) \frac{u_{j}\left(a_{i}\right) c_{3}}{\left(1+\lambda^{2} G_{j}^{\frac{2}{2-n}}\left(a_{i}\right)\right)^{\frac{n-2}{2}}}+o_{\varepsilon_{i, j}}\left(\varepsilon_{i, j}\right) \\
& +\int_{\mathcal{A}^{c} \cap B^{\lambda}}\left(\frac{1}{1+|x|^{2}}\right)^{\frac{n}{2}}\left[\left(\frac{1}{1+\lambda^{2} G_{j}^{\frac{2}{2-n}}}\right)^{\frac{n-2}{2}}\right] \circ \exp _{i}\left(\frac{x}{\lambda}\right) d x .
\end{aligned}
$$


Next, using (14), (39) and Taylor expansion, we derive that

$$
\begin{aligned}
\frac{u_{j}\left(a_{i}\right) c_{3}}{\left(1+\lambda^{2} G_{j}^{\frac{2}{2-n}}\left(a_{i}\right)\right)^{\frac{n-2}{2}}} & =c_{3}\left(1+o_{\varepsilon_{i, j}}(1)\right) u_{j}\left(a_{i}\right) \frac{G_{j}\left(a_{i}\right)}{\lambda^{n-2}} \\
& =c_{3}\left(1+o_{\varepsilon_{i, j}}(1)\right) \frac{G\left(a_{i}, a_{j}\right)}{\lambda^{n-2}}=\left(1+o_{\varepsilon_{i, j}}(1)\right) \varepsilon_{i, j}
\end{aligned}
$$

Thus, combining (38), (39), (41) and (42), we obtain

$$
\epsilon_{i, j}=\left(1+o_{\varepsilon_{i, j}}(1)\right) \varepsilon_{i, j}+I_{\mathcal{A}^{c}},
$$

where

$$
I_{\mathcal{A}^{c}}=\int_{\mathcal{A}^{c} \cap B^{\lambda}(0)}\left(\frac{1}{1+|x|^{2}}\right)^{\frac{n}{2}}\left[\left(\frac{1}{1+\lambda^{2} G_{j}^{\frac{2}{2-n}}}\right)^{\frac{n-2}{2}}\right] \circ \exp _{i}\left(\frac{x}{\lambda}\right) d x,
$$

and $\mathcal{A}^{c}=\mathbb{R}^{n-1} \backslash \mathcal{A}$. Hence, to end the proof of ii) of point 1) and to prove iii) of point 1 ), we are going to show that $I_{\mathcal{A}^{c}}$ satisfies

$$
I_{\mathcal{A}^{c}}=o_{\varepsilon_{i, j}}\left(\varepsilon_{i, j}\right) .
$$

In order to do that, we first decompose $\mathcal{A}^{c}$ into

$$
\mathcal{B}=\left\{x \in \mathbb{R}^{n-1}: \gamma \lambda \sqrt{G_{j}^{\frac{2}{2-n}}\left(a_{i}\right)} \leq|x| \leq \gamma^{-1} \lambda \sqrt{G_{j}^{\frac{2}{2-n}}\left(a_{i}\right)}\right\}
$$

and

$$
\mathcal{C}=\left\{x \in \mathbb{R}^{n-1}:|x|>\gamma^{-1} \lambda \sqrt{G_{j}^{\frac{2}{2-n}}\left(a_{i}\right)}\right\}
$$

and have

$$
I_{\mathcal{A}^{c}}=I_{\mathcal{B}}+I_{\mathcal{C}}
$$

where

(45) $I_{\mathcal{B}}:=\int_{\mathcal{B} \cap B^{\lambda}(0)}\left(\frac{1}{1+|x|^{2}}\right)^{\frac{n}{2}}\left[\left(\frac{1}{1+\lambda^{2} G_{j}^{\frac{2}{2-n}}}\right)^{\frac{n-2}{2}}\right] \circ \exp _{i}\left(\frac{x}{\lambda}\right) d x$,

and

(46) $I_{\mathcal{C}}:=\int_{\mathcal{C} \cap B^{\lambda}(0)}\left(\frac{1}{1+|x|^{2}}\right)^{\frac{n}{2}}\left[\left(\frac{1}{1+\lambda^{2} G_{j}^{\frac{2}{2-n}}}\right)^{\frac{n-2}{2}}\right] \circ \exp _{i}\left(\frac{x}{\lambda}\right) d x$. 
To prove (43), we are going to estimate separately $I_{\mathcal{B}}$ and $I_{\mathcal{C}}$. We start with $I_{\mathcal{B}}$. Using (44) and (45), we have clearly that $I_{\mathcal{B}}$ verifies the following estimate:

$$
I_{\mathcal{B}} \leq \frac{C_{\gamma}}{\left(1+\lambda^{2} G_{j}^{\frac{2}{2-n}}\left(a_{i}\right)\right)^{\frac{n}{2}}} \times \int_{\mathcal{B} \cap B^{\lambda}}\left[\left(\frac{1}{1+\lambda^{2} G_{j}^{\frac{2}{2-n}}}\right)^{\frac{n-2}{2}}\right] \circ \exp _{i}\left(\frac{x}{\lambda}\right) d x
$$

for some large positive constant $C_{\gamma}$ depending only on $\gamma$. Thus, rescaling and changing coordinates via $\exp _{j} \circ \exp _{i}^{-1}$ (if necessary), we have that (47) implies

$$
I_{\mathcal{B}} \leq \hat{C}_{\gamma} \varepsilon_{i, j}^{\frac{n}{n-2}} \int_{\left[|x| \leq \lambda \tilde{C}_{\gamma} d_{\hat{g}}\left(a_{i}, a_{j}\right)\right]}\left(\frac{1}{1+|x|^{2}}\right)^{\frac{n-2}{2}} d x \leq \bar{C}_{\gamma} \varepsilon_{i, j}^{\frac{n-1}{n-2}}
$$

for some large positive constants $\hat{C}_{\gamma}, \tilde{C}_{\gamma}$ and $\bar{C}_{\gamma}$ which are depending only on $\gamma$. Finally, we estimate $I_{\mathcal{C}}$. To do that, we fix $\gamma>0$ sufficiently small and use (38), (39) and (46) to obtain

$$
I_{\mathcal{C}} \leq \frac{C_{\gamma}}{\left(1+\lambda^{2} G_{j}^{\frac{2}{2-n}}\left(a_{i}\right)\right)^{\frac{n-2}{2}}} \int_{\mathcal{C}}\left(\frac{1}{1+|x|^{2}}\right)^{\frac{n}{2}} d x=o_{\varepsilon_{i, j}}\left(\varepsilon_{i, j}\right)
$$

for some large constant $C_{\gamma}$ depending only on $\gamma$. Hence (48) and (49) imply (43), thereby ending the proof of point 1 ). On the other hand, we have clearly that point 2) follows from Lemma 2.6 and Lemma 2.7. Furthermore, the first equation of point 3) follows from Lemma 2.6 and ii) of point 1), while the second equation follows from the first equation and from the self-adjointness of $\left(L_{g}, B_{g}\right)$.

q.e.d.

Now, using (12), (28), (29)-(31), we have the following interaction type estimate:

Lemma 2.9. Assuming that $p \in \mathbb{N}^{*}, A:=\left(a_{1}, \cdots, a_{p}\right) \in(\partial M)^{p}$ and $\lambda \geq \frac{1}{\delta_{0}^{n}}$, then for every $i, j=1, \cdots, p$ with $i \neq j$, there holds

$$
\oint_{\partial M} \varphi_{a_{i}, \lambda}^{\frac{n-1}{n-2}} \varphi_{a_{j}, \lambda}^{\frac{n-1}{n-2}} d S_{g}=O_{A_{i, j}, \lambda}\left(\varepsilon_{i, j}^{\frac{n-1}{n-2}} \log \varepsilon_{i, j}\right),
$$

where $A_{i, j}=\left(a_{i}, a_{j}\right), \varepsilon_{i, j}:=\varepsilon_{i, j}(A, \bar{\lambda})$ with $\bar{\lambda}:=(\lambda, \cdots, \lambda)$, and for the meaning of $O_{A_{i, j}, \lambda}(1)$ and $\epsilon_{i, j}(A, \bar{\lambda})$, see, respectively, Section 2 and (18).

Proof. Using (12), (28), (29)-(31) and setting $\varphi_{i}=\varphi_{a_{i}, \lambda}$ for $i=$ $1, \cdots, p$, we have that for every $i=1, \cdots, p$, the following estimate 
holds:

$$
\varphi_{i} \leq C\left(\frac{\lambda}{1+\lambda^{2} d_{\hat{g}}^{2}\left(a_{i}, \cdot\right)}\right)^{\frac{n-2}{2}} \text { on } \partial M
$$

for some large positive constant independent of $a_{i}$ and $\lambda$ with $\hat{g}$ denoting the Riemannian metric induced by $g$ on $\partial M$. Hence, using (51), we have for $c>0$ and small that the following estimate holds:

$$
\begin{aligned}
\oint_{\partial M} \varphi_{i}^{\frac{n-1}{n-2}} \varphi_{j}^{\frac{n-1}{n-2}} d S_{g} \\
\leq C \int_{B_{0}^{\mathbb{R}^{n-1}}(c)}\left(\frac{\lambda}{1+\lambda^{2}|x|^{2}}\right)^{\frac{n-1}{2}}\left(\frac{\lambda}{1+\lambda^{2} d_{\hat{g}}^{2}\left(a_{j}, \exp _{i}(x)\right)}\right)^{\frac{n-1}{2}} d x \\
+C \frac{1}{\lambda^{\frac{n-1}{2}}} \int_{B_{0}^{\mathbb{R}^{n-1}}(c)}\left(\frac{\lambda}{1+\lambda^{2}|x|^{2}}\right)^{\frac{n-1}{2}} d x+O_{A_{i, j}, \lambda}\left(\frac{1}{\lambda^{n-1}}\right) \\
=C \int_{B_{0}^{\mathbb{R} n^{n-1}}(c \lambda)}\left(\frac{1}{1+r^{2}}\right)^{\frac{n-1}{2}}\left(\frac{1}{1+\lambda^{2} d_{\hat{g}}^{2}\left(a_{j}, \exp _{i}\left(\frac{x}{\lambda}\right)\right)}\right)^{\frac{n-1}{2}} d x \\
+C \frac{1}{\lambda^{n-1}} \int_{B_{0}^{\mathbb{R}^{n-1}}(c \lambda)}\left(\frac{1}{1+|x|^{2}}\right)^{\frac{n-1}{2}} d x+O_{A_{i, j}, \lambda}\left(\frac{1}{\lambda^{n-1}}\right)
\end{aligned}
$$

for some large positive constant $C$ independent of $A_{i, j}$ and $\lambda$ with $r=|x|$ and $\exp _{i}:=\exp _{a_{i}}$ (for its meaning see Section 2). Thus appealing to (52), we infer that

$$
\begin{aligned}
\oint_{\partial M} \varphi_{i}^{\frac{n-1}{n-2}} & \varphi_{j}^{\frac{n-1}{n-2}} d S_{g} \\
\leq & C \int_{B_{0}^{\mathbb{R}^{n-1}}(c \lambda)}\left(\frac{1}{1+|x|^{2}}\right)^{\frac{n-1}{2}}\left(\frac{1}{1+\lambda^{2} d_{\hat{g}}^{2}\left(a_{j}, \exp _{i}\left(\frac{x}{\lambda}\right)\right)}\right)^{\frac{n-1}{2}} d x \\
& +O_{A_{i, j}, \lambda}\left(\frac{\log \lambda}{\lambda^{n-1}}\right) .
\end{aligned}
$$

Thus (50) follows from (53) if

$$
d_{\hat{g}}\left(a_{i}, a_{j}\right) \geq 3 c .
$$


Hence, to complete the proof of the lemma it remains to treat the case $d_{\hat{g}}\left(a_{i}, a_{j}\right)<3 c$. To do that, we set

$$
\mathcal{B}=\left\{x \in \overline{\mathbb{R}}^{n-1}: \quad \frac{1}{2} d_{\hat{g}}\left(a_{i}, a_{j}\right) \leq\left|\frac{x}{\lambda}\right| \leq 2 d_{\hat{g}}\left(a_{i}, a_{j}\right)\right\}
$$

and use (53) and the triangle inequality to get for $c>0$ sufficiently small that the following estimate holds:

$$
\begin{aligned}
& \oint_{\partial M} \varphi_{i}^{\frac{n-1}{n-2}} \varphi_{j}^{\frac{n-1}{n-2}} d S_{g} \\
& \leq C \int_{\mathcal{B}}\left(\frac{1}{1+|x|^{2}}\right)^{\frac{n-1}{2}}\left(\frac{1}{1+\lambda^{2} d_{\hat{g}}^{2}\left(a_{j}, \exp _{i}\left(\frac{x}{\lambda}\right)\right)}\right)^{\frac{n-1}{2}} d x \\
&+O_{A_{i, j}, \lambda}\left(\varepsilon_{i, j}^{\frac{n-1}{n-2}} \log \varepsilon_{i, j}\right) \\
& \leq C\left(\frac{1}{1+\left|\lambda d_{\hat{g}}\left(a_{i}, a_{j}\right)\right|^{2}}\right)^{\frac{n-1}{2}} \int_{\left\{\left|\frac{x}{\lambda}\right| \leq 4 d_{\hat{g}}\left(a_{i}, a_{j}\right)\right\}}\left(\frac{1}{1+|x|^{2}}\right)^{\frac{n-1}{2}} d x \\
&+O_{A_{i, j}, \lambda\left(\varepsilon_{i, j}^{\frac{n-1}{n-2}} \log \varepsilon_{i, j}\right)} \\
&= O_{A_{i, j}, \lambda}\left(\varepsilon_{i, j}^{\frac{n-1}{n-2}} \log \varepsilon_{i, j}\right),
\end{aligned}
$$

where $C$ is a large positive constant independent of $A_{i, j}$ and $\lambda$, thereby completing the proof of the lemma.

q.e.d.

\section{Energy estimates for the barycenter technique}

In this section, we map $B_{p}(\partial M)$ into some appropriate sublevels of the Euler-Lagrange functional $\mathcal{E}_{g}$ via the Almaraz[3]-Chen[34]'s bubbles. Precisely, we are going to derive sharp energy estimates for convex combinations of the bubbles $\varphi_{a, \lambda}$ given by (28) so that we can use them in the next section to run a suitable scheme of the barycenter technique of Bahri-Coron[14]. In order to do that, we first make the following definition. For $p \in \mathbb{N}^{*}, \sigma:=\sum_{i=1}^{p} \alpha_{i} \delta_{a_{i}} \in B_{p}(\partial M)$ and $\lambda \geq \frac{1}{\delta_{0}^{n}}$, where $\delta_{0}$ is given by Lemma 2.2, we define $f_{p}(\lambda): B_{p}(\partial M) \longrightarrow$ $W_{+}^{1,2}(\bar{M})$ as follows:

$$
f_{p}(\lambda)(\sigma):=\sum_{i=1}^{p} \alpha_{i} \varphi_{a_{i}, \lambda} .
$$

We start this section with the following proposition which provides the first step to apply our scheme of the algebraic topological argument of Bahri-Coron[14].

Proposition 3.1. There exists a large constant $C_{0}>0, \nu_{0}>1$ and $0<\varepsilon_{0} \leq \delta_{0}$ such that for every $p \in \mathbb{N}^{*}, p \geq 2$ and every $0<\varepsilon \leq \varepsilon_{0}$, there exists $\lambda_{p}:=\lambda_{p}(\varepsilon):=\lambda_{p}\left(\nu_{0}, \varepsilon\right) \geq \frac{1}{\delta_{0}^{n}}$ such that for every $\lambda \geq \lambda_{p}$ 
and for every $\sigma=\sum_{i=1}^{p} \alpha_{i} \delta_{a_{i}} \in B_{p}(\partial M)$, we have

1) If there exist $i_{0} \neq j_{0}$ such that $\frac{\alpha_{i_{0}}}{\alpha_{j_{0}}}>\nu_{0}$ or if $\sum_{i \neq j} \varepsilon_{i, j}>\varepsilon$, then

$$
\mathcal{E}_{g}\left(f_{p}(\lambda)(\sigma)\right) \leq p^{\frac{1}{n-1}} \mathcal{Q}\left(B^{n}\right),
$$

where $\mathcal{Q}\left(B^{n}\right)$ is defined by $(8), \varepsilon_{i, j}:=\varepsilon_{i, j}(A, \bar{\lambda})$ with $\bar{\lambda}:=(\lambda, \cdots, \lambda)$ and for the definition of $\varepsilon(A, \bar{\lambda})$, see (18).

2) If for every $i \neq j$ we have $\frac{\alpha_{i}}{\alpha_{j}} \leq \nu_{0}$ and if $\sum_{i \neq j} \varepsilon_{i, j} \leq \varepsilon$, then

$$
\mathcal{E}_{g}\left(f_{p}(\lambda)(\sigma)\right) \leq p^{\frac{1}{n-1}} \mathcal{Q}\left(B^{n}\right)\left(1+\frac{C_{0}}{\lambda^{n-2}}-c_{g} \frac{(p-1)}{\lambda^{n-2}}\right),
$$

where $c_{g}$ is defined by (16).

As in [57], Proposition 3.1 will be derived from the following technical lemma:

Lemma 3.2. We have that the following holds:

1) For every $\epsilon>0$ and small and for every $p \in \mathbb{N}^{*}$ with $p \geq 2$, there exists $\lambda_{p}:=\lambda_{p}(\epsilon) \geq \frac{1}{\delta_{0}^{n}}$ such that for every $\lambda \geq \lambda_{p}$ and for every $\sigma:=\sum_{i=1}^{p} \alpha_{i} \delta_{a_{i}} \in B_{p}(\partial M)$, we have that

$$
\sum_{i \neq j} \epsilon_{i, j}>\epsilon
$$

implies

$$
\mathcal{E}_{g}\left(f_{p}(\lambda)(\sigma)\right)<p^{\frac{1}{n-1}} \mathcal{Q}\left(B^{n}\right),
$$

where $\epsilon_{i, j}:=\epsilon_{i, j}(A, \lambda)$ is defined by $(32)$.

2) For every $\nu>1$, for every $\epsilon>0$ and small and for every $p \in \mathbb{N}^{*}$ with $p \geq 2$, there exists $\lambda_{p}:=\lambda_{p}(\epsilon, \nu) \geq \frac{1}{\delta_{0}^{n}}$ such that for every $\lambda \geq \lambda_{p}$ and for every $\sigma:=\sum_{i}^{p} \alpha_{i} \delta_{a_{i}} \in B_{p}(\partial M)$, we have

$$
\exists i_{0} \neq j_{0} \text { such that } \frac{\alpha_{i_{0}}}{\alpha_{j_{0}}}>\nu \quad \text { and } \quad \sum_{i \neq j} \epsilon_{i, j} \leq \epsilon
$$

imply

$$
\mathcal{E}_{g}\left(f_{p}(\lambda)(\sigma)\right)<p^{\frac{1}{n-1}} \mathcal{Q}\left(B^{n}\right) .
$$

3) There exists $C_{0}>0, \nu_{0}>1, \lambda_{0} \geq \frac{1}{\delta_{0}^{n}}$ and $0<\epsilon_{0} \leq \delta_{0}$ such that for every $1<\nu \leq \nu_{0}$, for every $0<\epsilon \leq \epsilon_{0}$, for every $p \in \mathbb{N}^{*}$ with $p \geq 2$, for every $\lambda \geq \lambda_{0}$ and for every $\sigma:=\sum_{i=1}^{p} \alpha_{i} \delta_{a_{i}} \in B_{p}(\partial M)$, we have

$$
\frac{\alpha_{i}}{\alpha_{j}} \leq \nu \quad \forall i, j, \quad \text { and } \quad \sum_{i \neq j} \epsilon_{i, j} \leq \epsilon
$$

imply

$$
\mathcal{E}_{g}\left(f_{p}(\lambda)(\sigma)\right) \leq p^{\frac{1}{n-1}} \mathcal{Q}(B)\left(1+\frac{C_{0}}{\lambda^{n-2}}-c_{g} \frac{(p-1)}{\lambda^{n-2}}\right)
$$


Proof. The strategy of the proof is the same as the one of Lemma 3.2 in [57]. For the sake of completeness we will provide full details. First of all, we set

$$
\mathcal{N}_{g}(u):=\left\langle L_{g} u, u\right\rangle+\left\langle B_{g} u, u\right\rangle, \mathcal{D}_{g}(u):=\left(\oint_{\partial M} u^{\frac{2(n-1)}{n-2}} d S_{g}\right)^{\frac{n-2}{n-1}},
$$

for $u \in W_{+}^{1,2}(\bar{M})$ and use (1) to have

$$
\mathcal{E}_{g}(u)=\frac{\mathcal{N}_{g}(u)}{\mathcal{D}_{g}(u)}, \quad u \in W_{+}^{1,2}(\bar{M}) .
$$

Furthermore, for $p \in \mathbb{N}^{*}, \sigma:=\sum_{i=1}^{p} \alpha_{i} \delta_{a_{i}} \in B_{p}(\partial M)$ and $\lambda \geq \frac{1}{\delta_{0}^{n}}$, we set (as in the proof of Lemma 2.8)

$$
\varphi_{i}=\varphi_{a_{i}, \lambda}, \quad i=1, \cdots, p .
$$

Now, we start with the proof of point 1). To do so, we first use Lemma $2.8,(54),(55),(57)$ and Hölder's inequality to estimate $\mathcal{N}_{g}\left(f_{p}(\lambda)(\sigma)\right)$ as follows:

$$
\begin{gathered}
\mathcal{N}_{g}\left(f_{p}(\lambda)(\sigma)\right)=c_{0}\left(1+o_{A, \bar{\alpha}, \lambda}(1)\right) \oint_{\partial M}\left(\sum_{i=1}^{p} \alpha_{i} \varphi_{i}^{\frac{n}{n-2}}\right)\left(\sum_{j=1}^{p} \alpha_{j} \varphi_{j}\right) d S_{g} \\
=c_{0}\left(1+o_{A, \bar{\alpha}, \lambda}(1)\right) \oint_{\partial M}\left(\frac{\sum_{i=1}^{p} \alpha_{i} \varphi_{i}^{\frac{n}{n-2}}}{\sum_{j=1}^{p} \alpha_{j} \varphi_{j}}\right)\left(\sum_{j=1}^{p} \alpha_{j} \varphi_{j}\right)^{2} d S_{g} \\
\leq c_{0}\left(1+o_{A, \bar{\alpha}, \lambda}(1)\right) \mathcal{D}_{g}\left(f_{p}(\lambda)(\sigma)\right)\left\|\frac{\sum_{i=1}^{p} \alpha_{i} \varphi_{i}^{\frac{n}{n-2}}}{\sum_{j=1}^{p} \alpha_{j} \varphi_{j}}\right\|_{L^{n-1}(\partial M)},
\end{gathered}
$$

where $A:=\left(a_{1}, \cdots, a_{p}\right), \bar{\alpha}:=\left(\alpha_{1}, \cdots, \alpha_{p}\right)$ and for the meaning of $o_{A, \bar{\alpha}, \lambda}(1)$, see Section 2 . Thus, using the convexity of the map $x \longrightarrow x^{\beta}$ with $\beta>1$, we derive that (58) implies

$$
\begin{aligned}
& \mathcal{N}_{g}\left(f_{p}(\lambda)(\sigma)\right) \leq c_{0}\left(1+o_{A, \bar{\alpha}, \lambda}(1)\right) \mathcal{D}_{g}\left(f_{p}(\lambda)(\sigma)\right)\left(\oint_{M}\left(\sum_{i=1}^{p} \frac{\alpha_{i} \varphi_{i}}{\sum_{j=1}^{p} \alpha_{j} \varphi_{j}} \varphi_{i}^{\frac{2}{n-2}}\right)^{n-1} d S_{g}\right)^{\frac{1}{n-1}} \\
& \leq c_{0}\left(1+o_{A, \bar{\alpha}, \lambda}(1)\right) \mathcal{D}_{g}\left(f_{p}(\lambda)(\sigma)\right) \\
& \\
&\left(\sum_{i=1}^{p} \oint_{\partial M} \frac{\alpha_{i} \varphi_{i}}{\sum_{j=1}^{p} \alpha_{j} \varphi_{j}} \varphi^{\frac{2(n-1)}{n-2}} d S_{g}\right)^{\frac{1}{n-1}}
\end{aligned}
$$


Hence, clearly Lemma 2.7, (56) and (59) imply for any pair $i \neq j$ $(i, j=1, \cdots, p)$

$$
\begin{aligned}
\mathcal{E}_{g}\left(f_{p}(\lambda)(\sigma)\right) \leq & c_{0}\left(1+o_{A, \bar{\alpha}, \lambda}(1)\right) \\
& \left(c_{1}(p-1)+\oint_{\partial M} \frac{\alpha_{i} \varphi_{i}}{\alpha_{i} \varphi_{i}+\alpha_{j} \varphi_{j}} \varphi_{i}^{\frac{2(n-1)}{n-2}} d S_{g}\right)^{\frac{1}{n-1}} \\
\leq & c_{0}\left(1+o_{A, \bar{\alpha}, \lambda}(1)\right) \\
& \left(c_{1} p-\oint_{\partial M} \frac{\alpha_{j} \varphi_{j}}{\alpha_{i} \varphi_{i}+\alpha_{j} \varphi_{j}} \varphi_{i}^{\frac{2(n-1)}{n-2}} d S_{g}\right)^{\frac{1}{n-1}},
\end{aligned}
$$

and we may assume $\alpha_{i} \leq \alpha_{j}$ by symmetry. Now, we are going to estimate from below the quantity $\oint_{\partial M} \frac{\alpha_{j} \varphi_{j}}{\alpha_{i} \varphi_{i}+\alpha_{j} \varphi_{j}} \varphi_{i}^{\frac{2(n-1)}{n-2}} d S_{g}$. In order to do that, for $\gamma>0$, we set

$$
\mathcal{A}_{i, j}=\left\{x \in \partial M: \varphi_{i}(x) \geq \gamma\left(\frac{\alpha_{i}}{\alpha_{j}} \varphi_{i}(x)+\varphi_{j}(x)\right)\right\}
$$

and use (61) to have

$$
\begin{aligned}
& \oint_{\partial M} \frac{\alpha_{j} \varphi_{j} \varphi_{i}^{\frac{2(n-1)}{n-2}}}{\alpha_{i} \varphi_{i}+\alpha_{j} \varphi_{j}} d S_{g} \geq \oint_{\mathcal{A}_{i, j}} \frac{\varphi_{j}}{\frac{\alpha_{i}}{\alpha_{j}} \varphi_{i}+\varphi_{j}} \varphi_{i}^{\frac{2(n-1)}{n-2}} d S_{g} \\
& \geq \gamma \oint_{\mathcal{A}_{i, j}} \varphi_{i}^{\frac{n}{n-2}} \varphi_{j} d S_{g} \\
& =\gamma\left(\oint_{\partial M} \varphi_{i}^{\frac{n}{n-2}} \varphi_{j} d S_{g}-\oint_{\mathcal{A}_{i, j}^{c}} \varphi_{i}^{\frac{n}{n-2}} \varphi_{j} d S_{g}\right) \\
& \geq \gamma\left(\oint_{\partial M} \varphi_{i}^{\frac{n}{n-2}} \varphi_{j} d S_{g}-\gamma^{\frac{2}{n-2}} \oint_{\mathcal{A}_{i, j}^{c}}\left(\frac{\alpha_{i}}{\alpha_{j}} \varphi_{i}+\varphi_{j}\right)^{\frac{2}{n-2}} \varphi_{i} \varphi_{j} d S_{g}\right),
\end{aligned}
$$

where $\mathcal{A}_{i, j}^{c}:=\partial M \backslash \mathcal{A}_{i, j}$. Next, since $\frac{\alpha_{i}}{\alpha_{j}} \leq 1$, then appealing to (62), we infer that the following estimate holds:

$$
\begin{aligned}
& \oint_{\partial M} \frac{\alpha_{j} \varphi_{j} \varphi_{i}^{\frac{2(n-1)}{n-2}}}{\alpha_{i} \varphi_{i}+\alpha_{j} \varphi_{j}} d S_{g} \\
& \quad \geq \gamma\left(\oint_{\partial M} \varphi_{i}^{\frac{n}{n-2}} \varphi_{j} d S_{g}-C \gamma^{\frac{2}{n-2}} \oint_{\partial M}\left(\varphi_{i}^{\frac{2}{n-2}}+\varphi_{j}^{\frac{2}{n-2}}\right) \varphi_{i} \varphi_{j} d S_{g}\right)
\end{aligned}
$$

for some large positive constant $C$ independent of $A, \lambda$ and $\gamma$. Thus, ii) of point 1) and point 3) of Lemma 2.8 together with (63) imply that for $\gamma>0$ sufficiently small, there holds

$$
\oint_{\partial M} \frac{\alpha_{j} \varphi_{j}}{\alpha_{i} \varphi_{i}+\alpha_{j} \varphi_{j}} \varphi_{i}^{\frac{2(n-1)}{n-2}} d S_{g} \geq \frac{\gamma}{2} \int_{\partial M} \varphi_{i}^{\frac{n}{n-2}} \varphi_{j} d S_{g} .
$$


Hence, combining (17), (60) and (64), we conclude that for any pair $i \neq j$, the following estimate holds:

$$
\begin{aligned}
& \mathcal{E}_{g}\left(f_{p}(\lambda)(\sigma)\right) \\
& \quad \leq\left(1+o_{A, \bar{\alpha}, \lambda}(1)\right) \mathcal{Q}\left(B^{n}\right)\left(p-\frac{\gamma}{2 c_{1}} \oint_{\partial M} \varphi_{i}^{\frac{n}{n-2}} \varphi_{j} d S_{g}\right)^{\frac{1}{n-1}} .
\end{aligned}
$$

Clearly (65) implies, that we always have

$$
\mathcal{E}_{g}\left(f_{p}(\lambda)(\sigma)\right) \leq\left(1+o_{A, \bar{\alpha}, \lambda}(1)\right) p^{\frac{1}{n-1}} \mathcal{Q}\left(B^{n}\right),
$$

and in case $\sum_{i \neq j} \epsilon_{i, j}>\epsilon$

$$
\mathcal{E}_{g}\left(f_{p}(\lambda)(\sigma)\right) \leq\left(1+o_{A, \bar{\alpha}, \lambda}(1)\right) p^{\frac{1}{n-1}} \mathcal{Q}\left(B^{n}\right)\left(1-\frac{\gamma \epsilon}{2 p c_{1}}\right)^{\frac{1}{n-1}},
$$

thereby ending the proof of point 1). Now, we are going to treat the second case. Hence, we may assume

$$
\sum_{i \neq j} \epsilon_{i, j} \ll 1
$$

and thus according to Lemma 2.8

$$
\epsilon_{i, j}=\left(1+o_{\varepsilon_{i, j}}(1)\right) \varepsilon_{i, j} \text { and } \lambda d_{\hat{g}}\left(a_{i}, a_{j}\right) \gg 1,
$$

and for the meaning of $o_{\epsilon_{i, j}}(1)$, see Section 2. We then use Lemma 2.8, (55) and (66) to have

$$
\begin{aligned}
\mathcal{N}_{g}( & \left.f_{p}(\lambda)(\sigma)\right)=\sum_{i=1}^{p} \sum_{j=1}^{p} \alpha_{i} \alpha_{j}\left(\left\langle L_{g} \varphi_{i}, \varphi_{j}\right\rangle+\left\langle B_{g} \varphi_{i}, \varphi_{j}\right\rangle\right) \\
= & \sum_{i=1}^{p} \alpha_{i}^{2}\left(\left\langle L_{g} \varphi_{i}, \varphi_{i}\right\rangle+\left\langle B_{g} \varphi_{i}, \varphi_{i}\right\rangle\right) \\
& \quad+\sum_{i \neq j} \alpha_{i} \alpha_{j}\left(\left\langle L_{g} \varphi_{i}, \varphi_{j}\right\rangle+\left\langle B_{g} \varphi_{i}, \varphi_{j}\right\rangle\right) \\
= & \sum_{i} \alpha_{i}^{2} \mathcal{E}_{g}\left(\varphi_{i}\right) \mathcal{D}_{g}\left(\varphi_{i}\right)+c_{0}\left(1+o_{\sum_{i, \neq j} \varepsilon_{i, j}}(1)\right) \sum_{i \neq j} \alpha_{i} \alpha_{j} \varepsilon_{i, j}
\end{aligned}
$$

and (for the meaning of $o_{\sum_{i \neq j} \epsilon_{i, j}}(1)$, see Section 2)

$$
\begin{aligned}
\mathcal{D}_{g}^{\frac{n-1}{n-2}}\left(f_{p}(\lambda)(\sigma)\right) & =\oint_{\partial M}\left(\sum_{i=1}^{p} \alpha_{i} \varphi_{i}\right)^{\frac{2(n-1)}{n-2}} d S_{g} \\
& =\sum_{i=1}^{p} \alpha_{i} \oint_{\partial M}\left(\sum_{j=1}^{p} \alpha_{j} \varphi_{j}\right)^{\frac{n}{n-2}} \varphi_{i} d S_{g}
\end{aligned}
$$




$$
=\sum_{i=1}^{p} \alpha_{i} \oint_{\partial M}\left(\alpha_{i} \varphi_{i}+\sum_{j=1, j \neq i}^{p} \alpha_{j} \varphi_{j}\right)^{\frac{n}{n-2}} \varphi_{i} d S_{g} .
$$

To proceed further, we set

$$
\mathcal{A}_{i}=\left\{x \in \partial M: \alpha_{i} \varphi_{i}(x)>\sum_{j=1, j \neq i}^{p} \alpha_{j} \varphi_{j}(x)\right\},
$$

and use Taylor expansion to obtain

$$
\begin{aligned}
\mathcal{D}_{g}^{\frac{n-1}{n-2}}\left(f_{p}(\lambda)(\sigma)\right)= & \sum_{i=1}^{p} \alpha_{i}^{\frac{2(n-1)}{n-2}} \oint_{\mathcal{A}_{i}} \varphi_{i}^{\frac{2(n-1)}{n-2}} d S_{g} \\
& +\frac{n}{n-2} \sum_{i \neq j} \alpha_{i}^{\frac{n}{n-2}} \alpha_{j} \oint_{\mathcal{A}_{i}} \varphi_{i}^{\frac{n}{n-2}} \varphi_{j} d S_{g} \\
& +\sum_{i=1}^{p} \alpha_{i} \oint_{\mathcal{A}_{i}^{c}}\left(\sum_{j=1, j \neq i}^{p} \alpha_{j} \varphi_{j}\right)^{\frac{n}{n-2}} \varphi_{i} d S_{g} \\
& +O_{A, \bar{\alpha}, \lambda}\left(\sum_{i \neq j} \alpha_{i}^{\frac{n-1}{n-2}} \alpha_{j}^{\frac{n-1}{n-2}} \oint_{\partial M} \varphi_{i}^{\frac{n-1}{n-2}} \varphi_{j}^{\frac{n-1}{n-2}} d S_{g}\right)
\end{aligned}
$$

where $\mathcal{A}_{i}^{c}:=\partial M \backslash \mathcal{A}_{i}, A:=\left(a_{1}, \cdots, a_{p}\right), \bar{\alpha}:=\left(\alpha_{1}, \cdots, \alpha_{p}\right), O_{A, \bar{\alpha}, \lambda}(1)$ is defined as in Section 2, and we made use of $n \geq 3$ and the algebraic relation

$$
(a+b)^{\frac{n-1}{n-2}} \leq C_{n}\left(a^{\frac{n-1}{n-2}}+b^{\frac{n-1}{n-2}}\right),
$$

for $a, b \geq 0$ and $C_{n}$ a positive constant depending only on $n$. Moreover, since

$$
(a+b)^{\frac{n}{n-2}} \geq a^{\frac{n}{n-2}}+b^{\frac{n}{n-2}}
$$

then (68) and (69) implies

$$
\begin{aligned}
\mathcal{D}_{g}^{\frac{n-1}{n-2}}\left(f_{p}(\lambda)(\sigma)\right) \geq & \sum_{i=1}^{p} \alpha_{i}^{\frac{2(n-1)}{n-2}} \oint_{\partial M} \varphi_{i}^{\frac{2(n-1)}{n-2}} d S_{g} \\
& +\frac{n}{n-2} \sum_{i \neq j} \alpha_{i}^{\frac{n}{n-2}} \alpha_{j} \oint_{\partial M} \varphi_{i}^{\frac{n}{n-2}} \varphi_{j} d S_{g} \\
& +\sum_{i \neq j} \alpha_{i} \alpha_{j}^{\frac{n}{n-2}} \oint_{A_{i}^{c}} \varphi_{j}^{\frac{n}{n-2}} \varphi_{i} d S_{g} \\
& +O_{A, \lambda}\left(\sum_{i \neq j} \alpha_{i}^{\frac{n-1}{n-2}} \alpha_{j}^{\frac{n-1}{n-2}} \oint_{\partial M} \varphi_{i}^{\frac{n-1}{n-2}} \varphi_{j}^{\frac{n-1}{n-2}} d S_{g}\right)
\end{aligned}
$$




$$
\begin{aligned}
\geq & \sum_{i=1}^{p} \alpha_{i}^{\frac{2(n-1)}{n-2}} \oint_{\partial M} \varphi_{i}^{\frac{2(n-1)}{n-2}} d S_{g} \\
& +2 \frac{n-1}{n-2} \sum_{i \neq j} \alpha_{i}^{\frac{n}{n-2}} \alpha_{j} \oint_{\partial M} \varphi_{i}^{\frac{n}{n-2}} \varphi_{j} d S_{g} \\
& +O_{A, \bar{\alpha}, \lambda}\left(\sum_{i \neq j} \alpha_{i}^{\frac{n-1}{n-2}} \alpha_{j}^{\frac{n-1}{n-2}} \oint_{\partial M} \varphi_{i}^{\frac{n-1}{n-2}} \varphi_{j}^{\frac{n-1}{n-2}} d S_{g}\right) .
\end{aligned}
$$

So, using Lemma 2.9 and (66), we have that (70) implies

$$
\begin{aligned}
\mathcal{D}_{g}^{\frac{n-1}{n-2}}\left(f_{p}(\lambda)(\sigma)\right) \geq & \sum_{i=1}^{p} \alpha_{i}^{\frac{2(n-1)}{n-2}} \oint_{\partial M} \varphi_{i}^{\frac{2(n-1)}{n-2}} d S_{g} \\
& +\frac{2(n-1)}{n-2}\left(1+o \sum_{i \neq j} \varepsilon_{i, j}(1)\right) \sum_{i \neq j} \alpha_{i}^{\frac{n}{n-2}} \alpha_{j} \varepsilon_{i, j} \\
& +o_{\sum_{i \neq j} \varepsilon_{i, j}}\left(\sum_{i \neq j} \alpha_{i}^{\frac{n-1}{n-2}} \alpha_{j}^{\frac{n-1}{n-2}} \varepsilon_{i, j}\right) .
\end{aligned}
$$

Thus, using Young's inequality and the symmetry of $\varepsilon_{i, j}$, we infer from (71) that the following estimate holds:

$$
\begin{aligned}
\mathcal{D}_{g}^{\frac{n-1}{n-2}}\left(f_{p}(\lambda)(\sigma)\right) \geq & \sum_{i=1}^{p} \alpha_{i}^{\frac{2(n-1)}{n-2}} \oint_{\partial M} \varphi_{i}^{\frac{2(n-1)}{n-2}} d S_{g} \\
& +\frac{2(n-1)}{n-2}\left(1+o_{\sum_{i \neq j} \varepsilon_{i, j}}(1)\right) \sum_{i \neq j} \alpha_{i}^{\frac{n}{n-2}} \alpha_{j} \varepsilon_{i, j}
\end{aligned}
$$

Hence, using again Young's inequality, Taylor expansion and Lemma 2.7 , we have that $(72)$ gives

$$
\begin{aligned}
\mathcal{D}_{g}\left(f_{p}(\lambda)(\sigma)\right) & \left(\sum_{i=1}^{p} \alpha_{i}^{\frac{2(n-1)}{n-2}} \oint_{\partial M} \varphi_{i}^{\frac{2(n-1)}{n-2}} d S_{g}\right)^{\frac{n-2}{n-1}} \\
& \left(1+\frac{\frac{2(n-1)}{n-2}\left(1+o_{\sum_{i \neq j} \varepsilon_{i, j}}(1)\right) \sum_{i \neq j} \alpha_{i}^{\frac{n}{n-2}} \alpha_{j} \varepsilon_{i, j}}{\sum_{i=1}^{p} \alpha_{i}^{\frac{2(n-1)}{n-2}} \oint_{\partial M} \varphi_{i}^{\frac{2(n-1)}{n-2}} d S_{g}}\right)^{\frac{n-2}{n-1}} \\
= & \left(\sum_{i=1}^{p} \alpha_{i}^{\frac{2(n-1)}{n-2}} \oint_{\partial M} \varphi_{i}^{\frac{2(n-1)}{n-2}} d S_{g}\right)^{\frac{n-2}{n-1}}
\end{aligned}
$$




$$
\begin{gathered}
+2 \frac{\left(1+o_{\sum_{i \neq j} \varepsilon_{i, j}}(1)\right) \sum_{i \neq j} \alpha_{i}^{\frac{n}{n-2}} \alpha_{j} \varepsilon_{i, j}}{\left(\sum_{i=1}^{p} \alpha_{i}^{\frac{2(n-1)}{n-2}} \oint_{\partial M} \varphi_{i}^{\frac{2(n-1)}{n-2}} d S_{g}\right)^{\frac{1}{n-1}}} \\
=\left(\sum_{i=1}^{p} \alpha_{i}^{\frac{2(n-1)}{n-2}} \mathcal{D}_{g}^{\frac{n-1}{n-2}}\left(\varphi_{i}\right)\right)^{\frac{n-2}{n-1}} \\
+2 c_{1}^{-\frac{1}{n-1}} \frac{\left(1+o \sum_{i \neq j} \varepsilon_{i, j}(1)\right) \sum_{i \neq j} \alpha_{i}^{\frac{n}{n-2}} \alpha_{j} \varepsilon_{i, j}}{\left(\sum_{i=1}^{p} \alpha_{i}^{\frac{2(n-1)}{n-2}}\right)^{\frac{1}{n-1}}}
\end{gathered}
$$

Now letting

$$
\mathcal{G}=\sum_{i=1}^{p} \alpha_{i}^{2} \mathcal{E}_{g}\left(\varphi_{i}\right) \mathcal{D}_{g}\left(\varphi_{i}\right)+c_{0}\left(1+o_{\sum_{i \neq j} \varepsilon_{i, j}}(1)\right) \sum_{i \neq j} \alpha_{i} \alpha_{j} \varepsilon_{i, j}
$$

and

$$
\begin{aligned}
\mathcal{H}= & \left(\sum_{i=1}^{p} \alpha_{i}^{\frac{2(n-1)}{n-2}} \mathcal{D}_{g}^{\frac{n-1}{n-2}}\left(\varphi_{i}\right)\right)^{\frac{n-2}{n-1}} \\
& +2 c_{1}^{-\frac{1}{n-1}} \frac{\left(1+o \sum_{i \neq j} \varepsilon_{i, j}(1)\right) \sum_{i \neq j} \alpha_{i}^{\frac{n}{n-2}} \alpha_{j} \varepsilon_{i, j}}{\left(\sum_{i=1}^{p} \alpha_{i}^{\frac{2(n-1)}{n-2}}\right)^{\frac{1}{n-1}}},
\end{aligned}
$$

we find from combining (67) and (73) via Taylor expansion

$$
\begin{aligned}
\mathcal{E}_{g}\left(f_{p}(\lambda)(\sigma)\right) \leq \frac{\mathcal{H}}{\mathcal{G}} \\
=\frac{\sum_{i=1}^{p} \mathcal{E}_{g}\left(\varphi_{i}\right) \alpha_{i}^{2} \mathcal{D}_{g}\left(\varphi_{i}\right)}{\left(\sum_{i=1}^{p} \alpha_{i}^{\frac{2(n-1)}{n-2}} \mathcal{D}_{g}^{\frac{n-1}{n-2}}\left(\varphi_{i}\right)\right)^{\frac{n-2}{n-1}}} \\
\quad+\frac{c_{0}}{c_{1}^{\frac{n-2}{n-1}}} \frac{\left(1+o \sum_{i \neq j} \varepsilon_{i, j}(1)\right) \sum_{i \neq j} \alpha_{i} \alpha_{j} \varepsilon_{i, j}}{\left(\sum_{i=1}^{p} \alpha_{i}^{\frac{2(n-1)}{n-2}}\right)^{\frac{n-2}{n-1}}} \\
\quad-\frac{2 c_{0}}{c_{1}^{\frac{n-2}{n-1}}} \frac{\left(1+o_{\sum_{i \neq j} \varepsilon_{i, j}}(1)\right)\left(\sum_{i=1}^{p} \alpha_{i}^{2}\right)\left(\sum_{i \neq j} \alpha_{i}^{\frac{n}{n-2}} \alpha_{j} \varepsilon_{i, j}\right)}{\left(\sum_{i=1}^{p} \alpha_{i}^{\frac{2(n-1)}{n-2}}\right)^{\frac{2 n-3}{n-1}}} .
\end{aligned}
$$


Hence, using (17) and rearranging the terms in (74), we get

$$
\begin{aligned}
& \mathcal{E}_{g}\left(f_{p}(\lambda)(\sigma)\right) \leq \max _{i=1, \cdots, p} \mathcal{E}_{g}\left(\varphi_{i}\right) \frac{\sum_{i=1}^{p} \alpha_{i}^{2} \mathcal{D}_{g}\left(\varphi_{i}\right)}{\left(\sum_{i=1}^{p}\left(\alpha_{i}^{2} \mathcal{D}_{g}\left(\varphi_{i}\right)\right)^{\frac{n-1}{n-2}}\right)^{\frac{n-2}{n-1}}} \\
& +\frac{\left(1+o \sum_{i \neq j} \varepsilon_{i, j}(1)\right) \mathcal{Q}\left(B^{n}\right)}{\left(\sum_{i=1}^{p} \alpha_{i}^{\frac{2(n-1)}{n-2}}\right)^{\frac{n-2}{n-1}}} \\
& \left(\sum_{i \neq j}\left[1-2 \frac{\alpha_{i}^{\frac{2}{n-2}}\left(\sum_{k=1}^{p} \alpha_{k}^{2}\right)}{\left(\sum_{k=1}^{p} \alpha_{k}^{\frac{2(n-1)}{n-2}}\right)}\right] \alpha_{i} \alpha_{j} \frac{\varepsilon_{i, j}}{c_{1}}\right) .
\end{aligned}
$$

This inequality has the following impact. First note for the first summand of (75), that the function

$$
\begin{aligned}
& \Gamma:\left\{\gamma \in[0,1]^{p}:\right.\left.\sum_{i=1}^{p} \gamma_{i}=1\right\} \longrightarrow \mathbb{R}_{+}, \\
& \gamma \longrightarrow \frac{\sum_{i=1}^{p} \gamma_{i}^{2}}{\left(\sum_{i=1}^{p} \gamma_{i}^{\frac{2(n-1)}{n-2}}\right)^{\frac{n-2}{n-1}}}
\end{aligned}
$$

has the strict global maximum

$$
\gamma_{\max }=\left(\frac{1}{p}, \ldots, \frac{1}{p}\right),
$$

with $\Gamma\left(\gamma_{\max }\right)=p^{\frac{1}{n-1}}$. Thus, using Lemma 2.7, Lemma 2.5, (55), (66) and (75), we infer that for any $\nu>0$, for every $\epsilon>0$ and small and for every $p \in \mathbb{N}^{*}$, there exists $\lambda_{p}:=\lambda_{p}(\nu, \epsilon) \geq \frac{1}{\delta_{0}^{n}}$ such for every $\lambda \geq \lambda_{p}$ and for every $\sigma:=\sum_{i=1}^{p} \alpha_{i} \delta_{a_{i}} \in B_{p}(\partial M)$, there holds

$$
\mathcal{E}_{g}\left(f_{p}(\lambda)(\sigma)\right)<p^{\frac{1}{n-1}} \mathcal{Q}\left(B^{n}\right)
$$

whenever

$$
\exists i_{o} \neq j_{0} \quad \text { such that } \quad \frac{\alpha_{i_{0}}}{\alpha_{j_{0}}}>\nu \quad \text { and } \quad \sum_{i \neq j} \varepsilon_{i, j} \leq \epsilon,
$$

thereby ending the proof of point 2). Now, we are going to treat point 3) and end the proof of the Lemma. Thus, we may assume

$$
\forall i, j \quad \frac{\alpha_{i}}{\alpha_{j}} \leq 1+o_{\mu}^{+}(1) \quad \text { and } \quad \sum_{i \neq j} \epsilon_{i, j} \ll 1,
$$

where $o_{\mu}^{+}(1)$ is a positive quantity depending only $\mu$ with $\mu$ small and verifying the property that it tends to 0 as $\mu$ tends to 0 . So, 
using (75), (78) and the properties of $\Gamma$ (see (76) and (77)), we infer that the following estimate holds:

$$
\begin{aligned}
& \mathcal{E}_{g}\left(f_{p}(\lambda)(\sigma)\right) \\
& \leq \max _{i=1, \cdots, p} \mathcal{E}_{g}\left(\varphi_{i}\right) p^{\frac{1}{n-1}} \\
&-\left(1+o_{\sum_{i \neq j} \varepsilon_{i, j}}(1)+o_{\mu}(1)\right) \frac{\mathcal{Q}\left(B^{n}\right) \sum_{i \neq j} \alpha_{i} \alpha_{j} \frac{\varepsilon_{i, j}}{c_{1}}}{\left(\sum_{i=1}^{p} \alpha_{i}^{\frac{2(n-1)}{n-2}}\right)^{\frac{n-2}{n-1}}} \\
&= \max _{i=1, \cdots, p} \mathcal{E}_{g}\left(\varphi_{i}\right) p^{\frac{1}{n-1}} \\
&-\left(1+o_{\sum_{i \neq j} \varepsilon_{i, j}}(1)+o_{\mu}(1)\right) \mathcal{Q}\left(B^{n}\right) p^{\frac{2-n}{n-1}} \sum_{i \neq j} \frac{\varepsilon_{i, j}}{c_{1}} .
\end{aligned}
$$

Now, using Lemma 2.5, (66), (78) and (79), we have that there exists $C_{0}>0, \nu_{0}>1, \lambda_{0} \geq \frac{1}{\delta_{0}^{n}}$ and $0<\epsilon_{0} \leq \delta_{0}$ such that for every $1<\nu \leq \nu_{0}$, for every $0<\epsilon \leq \epsilon_{0}$, for every $p \in \mathbb{N}^{*}$, for every $\lambda \geq \lambda_{0}$ and for every $\sigma:=\sum_{i=1}^{p} \alpha_{i} \delta_{a_{i}} \in B_{p}(\partial M)$, we have if $\frac{\alpha_{i}}{\alpha_{j}} \leq \nu \quad \forall i, j$ and $\sum_{i \neq j} \epsilon_{i, j} \leq \epsilon$, then there holds

$$
\mathcal{E}_{g}\left(f_{p}(\lambda)(\sigma)\right) \leq p^{\frac{1}{n-1}} \mathcal{Q}\left(B^{n}\right)\left(1+\frac{C_{0}}{\lambda^{n-2}}-\frac{1}{2 c_{1} p} \sum_{i \neq j} \varepsilon_{i, j}\right)
$$

Thus, recalling that (see $(42))$

$$
\varepsilon_{i, j}=\left(1+o_{\varepsilon_{i, j}}(1)\right) c_{3} \frac{G\left(a_{i}, a_{j}\right)}{\lambda^{n-2}}
$$

and using again (66), we infer from (80) that up to taking $\epsilon_{0}$ smaller, for every $1<\nu \leq \nu_{0}$, for every $0<\epsilon \leq \epsilon_{0}$, for every $p \in \mathbb{N}^{*}$, for every $\lambda \geq \lambda_{0}$ and for every $\sigma:=\sum_{i=1}^{p} \alpha_{i} \delta_{a_{i}} \in B_{p}(\partial M)$, there holds

$$
\frac{\alpha_{i}}{\alpha_{j}} \leq \nu \quad \forall i, j \quad \text { and } \quad \sum_{i \neq j} \epsilon_{i, j} \leq \epsilon
$$

imply

$$
\begin{aligned}
\mathcal{E}_{g}\left(f_{p}(\lambda)(\sigma)\right) & \leq p^{\frac{1}{n-1}} \mathcal{Q}\left(B^{n}\right)\left(1+\frac{C_{0}}{\lambda^{n-2}}-\frac{c_{3}}{4 c_{1} p \lambda^{n-2}} \sum_{i \neq j} G\left(a_{i}, a_{j}\right)\right) \\
& \leq p^{\frac{1}{n-1}} \mathcal{Q}\left(B^{n}\right)\left(1+\frac{C_{0}}{\lambda^{n-2}}-c_{g} \frac{(p-1)}{\lambda^{n-2}}\right),
\end{aligned}
$$

thereby ending the proof of point 3), hence of the Lemma.

q.e.d.

Proof of Proposition 3.1. It follows from Lemma 3.2 by taking $C_{0}$ and $\nu_{0}$ to be the ones given by Lemma 3.2, while $\varepsilon_{0}:=\frac{\epsilon_{0}}{2}$ and

$$
\lambda_{p}:=\lambda_{p}\left(\varepsilon, \nu_{0}\right):=\max \left\{\lambda_{p}\left(\frac{\varepsilon}{2}\right), \lambda_{p}\left(2 \varepsilon, \nu_{0}\right), \lambda_{0}\right\}
$$


where $\epsilon_{0}, \lambda_{p}\left(\frac{\varepsilon}{2}\right), \lambda_{p}\left(2 \varepsilon, \nu_{0}\right)$ and $\lambda_{0}$ are as in Lemma 3.2. $\quad$ q.e.d.

Now, using Proposition 3.1, we have the following corollary which will be used together with Proposition 3.1 in the next section to carry a suitable algebraic topological argument of Bahri-Coron[14].

Corollary 3.3. There exists $p_{0} \in \mathbb{N}^{*}$ large enough such that for every $0<\varepsilon \leq \varepsilon_{0}$ and for every for every $\lambda \geq \lambda_{p_{0}}$ (where $\varepsilon_{0}$ and $\lambda_{p_{0}}$ are given by Proposition 3.1), there holds

$$
f_{p_{0}}(\lambda)\left(B_{p_{0}}(\partial M)\right) \subset W_{p_{0}-1} .
$$

Proof. It follows directly from Proposition 3.1 and the definition of $W_{p_{0}-1}\left(\right.$ see $(7)$ with $p$ replaced by $\left.p_{0}-1\right)$.

q.e.d.

\section{Application of Bahri-Coron's barycenter technique}

In this section, we are going to use directly the results of the previous one to run a suitable scheme of the barycenter technique of BahriCoron[14]. To do so, we first introduce the notion of neighborhood of potential critical points at infinity of the Euler-Lagrange functional $\mathcal{E}_{g}$. Precisely, for $p \in \mathbb{N}^{*}, 0<\varepsilon \leq \varepsilon_{0}$ (where $\varepsilon_{0}$ is given by Proposition 3.1), we define $V(p, \varepsilon)$ the $(p, \varepsilon)$-neighborhood of potential critical points at infinity of $\mathcal{E}_{g}$, namely

$$
\begin{gathered}
V(p, \varepsilon):=\left\{u \in W^{1,2}(\bar{M}) \mid \exists a_{1}, \cdots, a_{p} \in \partial M, \alpha_{1}, \cdots, \alpha_{p}>0,\right. \\
\lambda_{1}, \cdots, \lambda_{p} \geq \frac{1}{\varepsilon}:\left\|u-\sum_{i=1}^{p} \alpha_{i} \varphi_{a_{i}, \lambda_{i}}\right\| \leq \varepsilon, \frac{\alpha_{i}}{\alpha_{j}} \leq \nu_{0} \\
\text { and for } \left.i \neq j: \varepsilon_{i, j} \leq \varepsilon\right\},
\end{gathered}
$$

where $\|\cdot\|$ denotes the standard $W^{1,2}$-norm, $\varepsilon_{i, j}:=\varepsilon_{i, j}(A, \bar{\lambda})$ with $A:=\left(a_{1}, \cdots, a_{p}\right), \bar{\lambda}:=\left(\lambda_{1}, \cdots, \lambda_{p}\right), \quad\left(\varepsilon_{i, j}(A, \bar{\lambda})\right)$ 's are defined by (18), $\varphi_{a_{i}, \lambda}$ is given by (28) for $i=1, \cdots, p$ and $\nu_{0}$ is given by Proposition 3.1.

Concerning the sets $V(p, \varepsilon)$, for every $p \in \mathbb{N}^{*}$, we have that there exists $0<\varepsilon_{p} \leq \varepsilon_{0}$ such that for every $0<\varepsilon \leq \varepsilon_{p}$, we have that

(81) $\forall u \in V(p, \varepsilon)$ the minimization problem $\min _{B_{C_{p} \varepsilon}}\left\|u-\sum_{i=1}^{p} \alpha_{i} \varphi_{a_{i}, \lambda_{i}}\right\|$ has a unique solution, up to permutations, where $B_{C_{p} \varepsilon}$ is defined as

$$
\begin{aligned}
& B_{C_{p} \varepsilon}:=\left\{(\bar{\alpha}, A, \bar{\lambda}) \in \mathbb{R}_{+}^{p} \times(\partial M)^{p} \times(0,+\infty)^{p} \mid\right. \\
&\left.\lambda_{i} \geq \frac{C_{p}}{\epsilon}, \frac{\alpha_{i}}{\alpha_{j}} \leq \nu_{0} \text { and for } i \neq j: \varepsilon_{i, j} \leq C_{p} \varepsilon p\right\}
\end{aligned}
$$

and $C_{p}>1$. Furthermore, we define the selection map

$$
s_{p}: V(p, \varepsilon) \longrightarrow(\partial M)^{p} / \sigma_{p},
$$


as follows:

$$
s_{p}(u):=A, \quad u \in V(p, \varepsilon), \text { and } A \text { is given by (81). }
$$

Now having introduced the neighborhoods of potential critical points at infinity of the Euler-Lagrange functional $\mathcal{E}_{g}$, we are ready to present our algebraic topological argument for existence. In order to do that, we start by the following classical deformation lemma which follows from the same arguments as for its counterparts in classical application of the algebraic topological argument of Bahri-Coron[14] (see, for example, Proposition 6 in [14]) and the fact that the $\varphi_{a, \lambda}$ can replace the standard bubbles in the analysis of diverging PS sequences of the Euler-Lagrange functional $\mathcal{E}_{g}$.

Lemma 4.1. Assuming that $\mathcal{E}_{g}$ has no critical points, then for every $p \in \mathbb{N}^{*}$, up to taking $\varepsilon_{p}$ smaller (where $\varepsilon_{p}$ is given by (81)), we have that for every $0<\varepsilon \leq \varepsilon_{p}$, there holds $\left(W_{p}, W_{p-1}\right)$ retracts by deformation onto $\left(W_{p-1} \cup \mathcal{A}_{p}, W_{p-1}\right)$ with $V(p, \tilde{\varepsilon}) \subset \mathcal{A}_{p} \subset V(p, \varepsilon)$ where $0<\tilde{\varepsilon}<\frac{\varepsilon}{4}$ is a very small positive real number and depends on $\varepsilon$.

Using Proposition 3.1 and Lemma 4.1, we are going to show that if $\mathcal{E}_{g}$ has no critical points, then for $\lambda$ large enough, the map $\left(f_{1}(\lambda)\right)_{*}$ is well defined and maps $\partial M$ (in top homology) in a nontrivial way in $\left(W_{1}, W_{0}\right)$. Precisely, we show:

Lemma 4.2. Assuming that $\mathcal{E}_{g}$ has no critical points and $0<\varepsilon \leq$ $\varepsilon_{1}$ (where $\varepsilon_{1}$ is given by (81)), then up to taking $\varepsilon_{1}$ smaller and $\lambda_{1}$ larger (where $\lambda_{1}$ is given by Proposition 3.1), we have that for every $\lambda \geq \lambda_{1}$

$$
f_{1}(\lambda):\left(B_{1}(\partial M), B_{0}(\partial M)\right) \longrightarrow\left(W_{1}, W_{0}\right)
$$

is well defined and satisfies

$$
\left(f_{1}(\lambda)\right)_{*}\left(w_{1}\right) \neq 0 \quad \text { in } \quad H_{n-1}\left(W_{1}, W_{0}\right) .
$$

Proof. It follows from the selection map $s_{1}$ given by (82), Lemma 2.5 and the same arguments as in Lemma 26 in [45].

q.e.d.

Next, as in [57], using Lemma 2.1, Proposition 3.1, Lemma 4.1 and the algebraic topological argument of Bahri-Coron[14], we are going to show that if for $\lambda$ large $B_{p}(\partial M)$ (in top homology) survives "topologically" the embedding into $\left(W_{p}, W_{p-1}\right)$ via $f_{p}(\lambda)$, then for $\lambda$ large $B_{p+1}(\partial M)$ (in top homology and as a cone with base $B_{p-1}(\partial M)$ and top $\partial M)$ survives "topologically" the embedding into $\left(W_{p+1}, W_{p}\right)$ via $f_{p+1}(\lambda)$. Precisely, we prove the following proposition:

Proposition 4.3. Assuming that $\mathcal{E}_{g}$ has no critical points and $0<\varepsilon \leq \varepsilon_{p+1}$ (where $\varepsilon_{p+1}$ is given by (81)), then up to taking $\varepsilon_{p+1}$ 
smaller and $\lambda_{p}$ and $\lambda_{p+1}$ larger (where $\lambda_{p}$ and $\lambda_{p+1}$ are given by Proposition 3.1), we have that for every $\lambda \geq \max \left\{\lambda_{p}, \lambda_{p+1}\right\}$, there holds

$$
f_{p+1}(\lambda):\left(B_{p+1}(\partial M), B_{p}(\partial M)\right) \longrightarrow\left(W_{p+1}, W_{p}\right),
$$

and

$$
f_{p}(\lambda):\left(B_{p}(\partial M), B_{p-1}(\partial M)\right) \longrightarrow\left(W_{p}, W_{p-1}\right)
$$

are well defined and satisfy

$$
\left(f_{p}(\lambda)\right)_{*}\left(w_{p}\right) \neq 0 \quad \text { in } \quad H_{n p-1}\left(W_{p}, W_{p-1}\right)
$$

implies

$$
\left(f_{p+1}(\lambda)\right)_{*}\left(w_{p+1}\right) \neq 0 \quad \text { in } \quad H_{n(p+1)-1}\left(W_{p+1}, W_{p}\right) .
$$

Proof. First of all, we let $p \in \mathbb{N}^{*}$ and $0<\varepsilon_{p+1}$, where $\varepsilon_{p+1}$ is given by (81). Next, recalling that we have assumed that $\mathcal{E}_{g}$ has no critical points, and using Lemma 4.1, then up to taking $\varepsilon_{p+1}$ smaller, we infer that the following holds:

$$
\left(W_{p+1}, W_{p}\right) \simeq\left(W_{p} \cup \mathcal{A}_{p+1}, W_{p}\right),
$$

with

$$
V(p+1, \tilde{\varepsilon}) \subset \mathcal{A}_{p+1} \subset V(p+1, \varepsilon), \quad 0<4 \tilde{\varepsilon}<\varepsilon .
$$

Now, using Lemma 2.8 and Proposition 3.1, we have that for every $\lambda \geq \max \left\{\lambda_{p}, \lambda_{p+1}\right\}$ (where $\lambda_{p}$ and $\lambda_{p+1}$ are given by Proposition 3.1), there holds

$$
f_{p+1}(\lambda):\left(B_{p+1}(\partial M), B_{p}(\partial M)\right) \longrightarrow\left(W_{p+1}, W_{p}\right),
$$

and

$$
f_{p}(\lambda):\left(B_{p}(\partial M), B_{p-1}(\partial M)\right) \longrightarrow\left(W_{p}, W_{p-1}\right)
$$

are well defined and hence have that the first point is proven. Next, using Proposition 3.1, (85) and (86), we have that up to taking $\lambda_{p+1}$ and $\lambda_{p}$ larger (for example, larger than $4 \max \left\{\lambda_{p+1}(\varepsilon), \lambda_{p}(\varepsilon), \lambda_{p}(2 \tilde{\varepsilon}), \lambda_{p}\left(\frac{\tilde{\varepsilon}}{2}\right)\right.$, $\left.\frac{1}{\tilde{\varepsilon}}\right\}$, where $\lambda_{p}(\varepsilon), \lambda_{p+1}(\varepsilon), \lambda_{p}(2 \tilde{\varepsilon})$, and $\lambda_{p}\left(\frac{\tilde{\varepsilon}}{2}\right)$ are given by Proposition 3.1 and $\tilde{\varepsilon}$ is given by (84)) the following diagram:

$$
\begin{array}{cc}
\left(B_{p+1}(\partial M), \mathcal{O}\left(B_{p}(\partial M)\right)\right) \stackrel{f_{p+1}(\lambda)}{\longrightarrow}\left(W_{p+1}, W_{p}\right) \\
\uparrow & \uparrow \\
\left(\mathcal{O}\left(B_{p}(\partial M)\right), B_{p-1}(\partial M)\right) \stackrel{f_{p}(\lambda)}{\longrightarrow}\left(W_{p}, W_{p-1}\right)
\end{array}
$$

is well defined and commutes, where

$$
\begin{aligned}
\mathcal{O}\left(B_{p}(\partial M)\right):=\{\sigma & =\sum_{i=1}^{p+1} \alpha_{i} \delta_{a_{i}} \in B_{p+1}(\partial M) \mid \\
& \left.\exists i_{0} \neq j_{0}: \frac{\alpha_{i_{0}}}{\alpha_{j_{0}}}>\nu_{0} \text { or } \sum_{i \neq j} \varepsilon_{i, j}>\tilde{\varepsilon}\right\},
\end{aligned}
$$


with $\nu_{0}$ given by Proposition 3.1. On the other hand, we have

$$
\mathcal{O}\left(B_{p}(\partial M)\right) \simeq B_{p}(\partial M)
$$

and

$$
B_{p+1}(\partial M) \backslash \mathcal{O}\left(B_{p}(\partial M)\right) \simeq B_{p+1}(\partial M) \backslash B_{p}(\partial M) .
$$

Now, using Lemma 2.1, we derive

$$
\begin{aligned}
& H^{n-1}\left((\partial M)^{p+1} / \sigma_{p+1}\right) \times H_{n(p+1)-1}\left(B_{p+1}(\partial M), B_{p}(\partial M)\right) \\
& \longrightarrow H_{n(p+1)-n}\left(B_{p+1}(\partial M), B_{p}(\partial M)\right) \\
& \stackrel{\partial}{\longrightarrow} H_{n(p+1)-n-1}\left(B_{p}(\partial M), B_{p-1}(\partial M)\right) .
\end{aligned}
$$

Furthermore, using (83), we infer that

$$
\begin{aligned}
H^{n-1}\left(\mathcal{A}_{p+1}\right) \times H_{n(p+1)-1}\left(W_{p+1}, W_{p}\right) & \\
\quad & \longrightarrow H_{n(p+1)-n}\left(W_{p+1}, W_{p}\right) \stackrel{\partial}{\longrightarrow} H_{n(p+1)-n-1}\left(W_{p}, W_{p-1}\right) .
\end{aligned}
$$

Moreover, passing to homologies in (87) and using (89), we derive

$$
\begin{aligned}
& H_{n(p+1)-1}\left(B_{p+1}(\partial M), B_{p}(\partial M)\right) \\
& \stackrel{\left(f_{p+1}(\lambda)\right)_{*}}{\longrightarrow} H_{n(p+1)-1}\left(W_{p+1}, W_{p}\right),
\end{aligned}
$$

and

$$
H_{n p-1}\left(B_{p}(\partial M), B_{p-1}(\partial M)\right) \stackrel{\left(f_{p}(\lambda)\right)_{*}}{\longrightarrow} H_{n p-1}\left(W_{p}, W_{p-1}\right)
$$

are well defined and the following diagram commutes:

$$
\begin{array}{ccc}
H_{n(p+1)-n}\left(B_{p+1}(\partial M), B_{p}(\partial M)\right) & \stackrel{\left(f_{p+1}(\lambda)\right)_{*}}{\longrightarrow} H_{n(p+1)-n}\left(W_{p+1}, W_{p}\right) \\
\partial \downarrow & & \\
H_{n p-1}\left(B_{p}(\partial M), B_{p-1}(\partial M)\right) & \stackrel{\left(f_{p}(\lambda)\right)_{*}}{\longrightarrow} & H_{n p-1}\left(W_{p}, W_{p-1}\right) .
\end{array}
$$

Next, recalling that we have taken $\lambda_{p+1}$ and $\lambda_{p}$ larger than

$$
4 \max \left\{\lambda_{p+1}(\varepsilon), \lambda_{p}(\varepsilon), \lambda_{p}(2 \tilde{\varepsilon}), \lambda_{p}\left(\frac{\tilde{\varepsilon}}{2}\right), \frac{1}{\tilde{\varepsilon}}\right\},
$$

we derive that

$$
\begin{aligned}
& f_{p+1}(\lambda)\left(B_{p+1}(\partial M) \backslash \mathcal{O}\left(B_{p}(\partial M)\right)\right) \\
& \subset V(p+1, \tilde{\varepsilon}) \subset \mathcal{A}_{p+1} \subset V(p+1, \varepsilon) .
\end{aligned}
$$

Thus, using (22), (82), (88), (89) and (94), we infer as in formula (6.3) in $[\mathbf{4 5}]$ that (see also $[\mathbf{1 2}]$ or $[\mathbf{1 4}]$ )

$$
\begin{aligned}
\left(f_{p+1}(\lambda)\right)^{*}\left(s_{p+1}^{*}\left(O_{\partial M}^{*}\right)\right) & =O_{\partial M}^{*}, \text { with } \\
s_{p+1}^{*}\left(O_{\partial M}^{*}\right) & \neq 0 \text { in } H^{n-1}\left(\mathcal{A}_{p+1}\right) .
\end{aligned}
$$


On the other hand, using (93), we derive that

$$
\partial\left(f_{p+1}(\lambda)\right)_{*}=\left(f_{p}(\lambda)\right)_{*} \partial \text { in } H_{n(p+1)-n}\left(B_{p+1}(\partial M), B_{p}(\partial M)\right) .
$$

Now, combining Lemma 2.1, (88), (90), (91), (92), (95) and (96), we obtain

$$
\begin{aligned}
\left(f_{p}(\lambda)\right)_{*}\left(\omega_{p}\right) & =\left(f_{p}(\lambda)\right)_{*}\left(\partial\left(O_{\partial M}^{*} \frown \omega_{p+1}\right)\right) \\
& =\left(f_{p}(\lambda)\right)_{*}\left(\partial\left(\left(\left(f_{p+1}(\lambda)\right)^{*}\left(s_{p+1}^{*}\left(O_{\partial M}^{*}\right)\right)\right) \frown \omega_{p+1}\right)\right) \\
& =\partial\left(\left(f_{p+1}(\lambda)\right)_{*}\left(\left(f_{p+1}(\lambda)\right)^{*}\left(s_{p+1}^{*}\left(O_{\partial M}^{*}\right)\right) \frown \omega_{p+1}\right)\right) \\
& =\partial\left(s_{p+1}^{*}\left(O_{\partial M}^{*}\right) \frown\left(\left(f_{p+1}(\lambda)\right)_{*}\left(\omega_{p+1}\right)\right)\right),
\end{aligned}
$$

with all the equalities holding in $H_{n p-1}\left(W_{p}, W_{p-1}\right)$. Hence, clearly (97) and the assumption

$$
\left(f_{p}(\lambda)\right)_{*}\left(w_{p}\right) \neq 0 \quad \text { in } \quad H_{n p-1}\left(W_{p}, W_{p-1}\right)
$$

imply

$$
\left(f_{p+1}(\lambda)\right)_{*}\left(w_{p+1}\right) \neq 0 \text { in } H_{n(p+1)-1}\left(W_{p+1}, W_{p}\right)
$$

as desired, thereby completing the proof of Proposition 4.3. q.e.d.

Now, we are ready to present the proof of Theorem 1.1.

Proof of Theorem 1.1. As in [57], it follows by a contradiction argument from Corollary 3.3, Lemma 4.2 and Proposition 4.3. q.e.d.

\section{References}

[1] M. Ahmedou \& C.B. Ndiaye, Morse theory and the resonant Q-curvature problem, arXiv:1409.7919 [math.DG].

[2] S.M. Almaraz, An existence theorem of conformal scalar flat metrics on manifolds with boundary, Pacific J. Math. 248 (2010), no. 1, 1-22, MR 2734161, Zbl 1205.53043.

[3] S.M. Almaraz, Convergence of scalar-flat metrics on manifolds with boundary under the Yamabe flow, J. Differential. Equations, 259 (2015), no 7, 2626-2694, MR 3360653, Zbl 1320.53078.

[4] A. Ambrosetti A, Y. Li \& A. Malchiodi, On the Yamabe problem and the scalar curvature problems under boundary conditions, Math. Ann. 322 (2002), no. 4, 667-699, MR 1905107, Zbl 1005.53034.

[5] A. Ambrosetti \& A. Malchiodi, A multiplicity result for the Yamabe problem on $S^{n}$, J. Funct. Anal. 168 (1999), no. 2, 529-561, MR 1719213, Zbl 0949.53028.

[6] H. Araujo, Critical points of the total scalar curvature plus total mean curvature functional, Indiana Univ. Math. J. 52 (2003), no. 1, 85-107, MR 1970022, Zbl 1050.53028 .

[7] H. Araujo, Existence and Compactness of Minimizers of the Yamabe problem on Manifolds with Boundary, Comm. Anal. Geom. (12) (2004), no 3, 487-510, MR2128601, Zbl 1070.53016.

[8] T. Aubin, Equations differentielles non lineaires et probleme de Yamabe concernant la courbure scalaire, J. Math. Pures Appl. (9) 55 (1976), no. 3, 269-296, MR 0431287, Zbl 0336.53033. 
[9] T. Aubin, Some nonlinear problems in Riemannian geometry, Springer Monographs in Mathematics, Springer-Verlag, Berlin 1998, MR 1636569, Zbl 0896.53003.

[10] T. Aubin \& A. Bahri, Methods of algebraic topology for the problem of prescribed scalar curvature. J. Math. Pures Appl. (9) 76 (1997), no. 6, 525-549, MR 1465609, Zbl 0886.58109.

[11] T. Aubin, \& A Bahri, A topological hypothesis for the problem of prescribed scalar curvature. J. Math. Pures Appl. (9) 76 (1997), no. 10, 843-850, MR 1489940, Zbl 0916.58041.

[12] A. Bahri, Critical points at infinity in some variational problems, Research Notes in Mathematics, 182, Longman-Pitman, London, 1989, MR 1019828, Zbl 0676.58021.

[13] A. Bahri, Another proof of the Yamabe conjecture for locally conformally flat manifolds, Nonlinear Anal. 20 (1993), no. 10, 1261-1278, MR 1219242, Zbl 0782.53027 .

[14] A. Bahri \& J.M. Coron, On a nonlinear elliptic equation involving the critical Sobolev exponent: the effect of the topology of the domain, Comm. Pure Appl. Math. 41-3 (1988), 253-294, MR 0929280, Zbl 0649.35033.

[15] A. Bahri \& J.M. Coron, The scalar-curvature problem on the standard threedimensional sphere, J. Funct. Anal. 95 (1991), no. 1, 106-172, MR 1087949, Zbl 0722.53032 .

[16] T.P. Branson \& B. Oersted, Explicit functional determinants in four dimensions, Proc. Amer. Math. Soc 113-3 (1991), 669-682, MR 1050018, Zbl 0762.47019.

[17] G.E. Bredon, Topology and geometry, Graduate Texts in Mathematics, 139, 1997.cand, 57-2 (1993), 293-345, MR 1224675, Zbl 0791.55001.

[18] M. Berti \& A. Malchiodi, Non-compactness and multiplicity results for the Yamabe problem on $S^{n}$, J. Funct. Anal. 180 (2001), no. 1, 210-241, MR 1814428, Zbl 0979.53038.

[19] S. Brendle, Global existence and convergence for a higher order flow in conformal geometry, Ann. of Math. 158 (2003), 323-343, MR 1999924, Zbl 1042.53016.

[20] S. Brendle, Convergence of the Yamabe flow for arbitrary initial energy, J. Diff. Geom. 69 (2005), 217-278, MR 2168505, Zbl 1085.53028.

[21] S. Brendle, Convergence of the Yamabe flow in dimension 6 and higher, Inv. math, 170 (2007), 541-576, MR 2357502, Zbl 1130.53044.

[22] S. Brendle, Blow-up phenomena for the Yamabe equation, J. Amer. Math. Soc. 21 (2008), no. 4, 951-979, MR 2425176, Zbl 1206.53041.

[23] S. Brendle \& S.Y.S. Chen, An existence theorem for the Yamabe problem on manifolds with boundary. J. Eur. Math. Soc. (JEMS) 16 (2014), no. 5, 991-1016, MR 3210959, Zbl 1293.53047.

[24] S. Brendle \& F.C. Marques, Blow-up phenomena for the Yamabe equation. II, J. Diff. Geom. 81 (2009), no. 2, 225-250, MR 2472174, Zbl 1166.53025.

[25] G. Catino \& C.B. Ndiaye, Integral pinching results for manifolds with boundary, Ann. Sc. Norm. Super. Pisa Cl. Sci. 9 (2010), 785-813, MR 2789475, Zbl 1246.53062 .

[26] A. Chang, M.J. Gursky, \& P.C. Yang. The scalar curvature equation on 2- and 3-spheres, Calc. Var. Partial Differential Equations 1 (1993), no. 2, 205-229, MR 1261723, Zbl 0822.35043. 
[27] S.Y.A. Chang, M.J Gursky, \& P.C. Yang, An equation of Monge-Ampere type in conformal geometry, and four-manifolds of positive Ricci curvature, Ann. of Math. 155-3 (2002), 709-787, MR 1923964, Zbl 1031.53062.

[28] S.Y.A Chang, M.J. Gursky, \& P.C. Yang, A conformally invariant sphere theorem in four dimensions, Publ. Math. Inst. Hautes etudes Sci. 989 (2003), 105143, MR 2031200Z, bl 06512738.

[29] S.Y.A Chang \& J. Qing, The Zeta Functional Determinants on manifolds with boundary 1. The Formula, Journal of Functional Analysis 147 (1997), 327-362, MR 1454485, Zbl 0914.58039.

[30] S.Y.A Chang \& J. Qing, The Zeta Functional Determinants on manifolds with boundary II. Extremal Metrics and Compactness of Isospectral Set, Journal of Functional Analysis 147 (1997), 363-399, MR 1454486, Zbl 0914.58040.

[31] S.Y.A Chang, J. Qing, \& P.C. Yang, Compactification of a class of conformally flat 4-manifold, Invent. Math. 142-1 (2000), 65-93, MR 1784799Zbl 0990.53026.

[32] S.Y.A Chang, J. Qing, \& P.C. Yang, On the Chern-Gauss-Bonnet integral for conformal metrics on $\mathbf{R}^{4}$, Duke Math. J. 03-3 (2000), 523-544, MR 1763657, Zbl 0971.53028.

[33] S.Y.A Chang \& P.C. Yang Extremal metrics of zeta functional determinants on 4-manifolds, Ann. of Math. 142 (1995), 171-212, MR 1338677, Zbl 0842.58011.

[34] S.Y.S. Chen, Conformal deformation to scalar flat metrics with constant mean curvature on the boundary in higher dimensions, arXiv:0912.1302.

[35] P. Cherrier, Problemes de Neumann non lineaires sur les varietes riemanniennes. (French) [Nonlinear Neumann problems on Riemannian manifolds], J. Funct. Anal. 57 (1984), no. 2, 15-206, MR 0749522, Zbl 0552.58032.

[36] Z. Djadli \& A. Malchiodi, Existence of conformal metrics with constant $Q$ curvature, Ann. of Math. (2) 168 (2008), no. 3, 813-858, MR 2456884, Zbl 1186.53050 .

[37] J.F. Escobar, The Yamabe problem on manifolds with boundary, Journal Differential Geometry 35 (1992) no. 1, 21-84, MR 1152225, Zbl 0771.53017.

[38] J.F Escobar, Conformal deformation of a Riemannian metric to a scalar flat metric with constant mean curvature on the boundary, Ann. of Math. (2) 136 (1992), no. 1, 1-50, MR 1173925, Zbl 0766.53033.

[39] J.F. Escobar, Conformal deformation of a Riemannian metric to a constant scalar curvature metric with constant mean curvature on the boundary, Indian. Univ. Mat. J. 45 (1996), 917-943, MR 1444473, Zbl 0881.53037.

[40] J.F Escobar, Conformal metrics with prescribed mean curvature on the boundary, Calc. Var. Partial Differential Equations 4 (1996), no. 6, 559-592, MR 1416000, Zbl 0867.53034.

[41] J.F. Escobar \& R. Scheon, Conformal metrics with prescribed scalar curvature, Invent. Math. 86 (1986), no. 2, 243-254, MR 0856845, Zbl 0628.53041.

[42] C. Fefferman C \& R. Graham, Conformal invariants, In Elie Cartan et les mathematiques d'aujourd'hui. Asterisque (1985), 95-116.

[43] C. Fefferman C \& R. Graham, Q-curvature and Poincaré metrics, Mathematical Research Letters 9 (2002), 139-151, MR 1909634, Zbl 1016.53031.

[44] N. Gamara, The CR Yamabe conjecture - the case $n=1$, J. Eur. Math. Soc. (JEMS) 3 (2001), no 2, 105-137, MR 1831872, Zbl 0988.53013. 
[45] N. Gamara \& R. Yacoub, CR Yamabe conjecture - the conformally flat case, Pacific J. Math. 201 (2001), no. 1, 121-175, MR 1867895, Zbl 1054.32020.

[46] D. Gilbarg \& N. Trudinger, Elliptic Partial Differential Equations of Second Order, 2nd edition, Springer-Verlag, 1983, MR 0737190, Zbl 0562.35001.

[47] M. Gursky \& J. Viaclovsky, A fully nonlinear equation on four-manifolds with positive scalar curvature, J. diff. Geom. 63 (2003), 131-154, MR 2015262Zbl, 1070.53018 .

[48] Z.C Han \& Y. Li, The Yamabe problem on manifolds with boundary: existence and compactness results, Duke Math. J. 99 (1999), no. 3, 489-542, MR 1712631, Zbl 0945.53023.

[49] Z.C Han \& Y. Li, The existence of conformal metrics with constant scalar curvature and constant boundary mean curvature, Comm. Anal. Geom. 8 (2000), no. 4, 809-869, MR 1792375, Zbl 0990.53033.

[50] S. Kallel \& R. Karoui R, Symmetric joins and weighted barycenters, Advanced Nonlinear Studies 11 (2011), 117-143, MR 2724545, Zbl 1228.55009.

[51] M.A. Khuri, F.C. Marques, \& R.M. Schoen, A compactness theorem for the Yamabe problem, J. Differential Geom. 81 (2009), no. 1, 143-196, MR 2477893, Zbl 1162.53029 .

[52] J. Lee \& T. Parker, The Yamabe problem, Bull. A.M.S. 17 (1987), 37-81, MR 0888880, Zbl 0633.53062.

[53] Y. Li, Prescribing scalar curvature on $S^{n}$ and related problems. I, J. Differential Equations 120 (1995), no. 2, 319-410, MR 1347349, Zbl 0827.53039.

[54] Y. Li, Prescribing scalar curvature on $S^{n}$ and related problems. II. Existence and compactness, Comm. Pure Appl. Math. 49 (1996), no. 6, 541-597, MR 1383201, Zbl 0849.53031.

[55] F.C. Marques, Existence results for the Yamabe problem on manifolds with boundary, Indiana Univ. Math. J. (2005), 1599-1620, MR 2189679, Zbl 1090.53043.

[56] F.C. Marques, Conformal deformations to scalar-flat metrics with constant mean curvature on the boundary, Comm. Anal. Geom. 15 (2007), no. 2, 381-405, MR 2344328, Zbl 1132.53021.

[57] M. Mayer \& C.B. Ndiaye, Proof of the remaining cases of the boundary Yamabe problem, https://arxiv.org/abs/1505.06114.

[58] C.B Ndiaye, Constant Q-curvature metrics in arbitrary dimension, J. Funct. Anal. 251 (2007), no. 1, 1-58. 53, MR 2353700, Zbl 1130.53027.

[59] C.B. Ndiaye, Conformal metrics with constant Q-curvature for manifolds with boundary, Comm. Anal. Geom. 16 (2008), no. 5, 1049-1124, MR 2471367, Zbl 1163.53324 .

[60] C.B. Ndiaye, Constant T-curvature conformal metric on 4-manifolds with boundary, Pacific J. Math. 240 (2009), no. 1, 151-184, MR 2485478, Zbl 1173.35009.

[61] C.B Ndiaye, Q-curvature flow on 4-manifolds with boundary, Math. Z. 269 (2011), 83-114, MR 2836061, Zbl 1238.53042.

[62] C.B. Ndiaye, Algebraic topological methods for the supercritical Q-curvature problem, Adv. Math 277 (2015), 56-99, MR 2836061, Zbl 1312.53062.

[63] C.B. Ndiaye \& J. Xiao J, An upper bound of the total Q-curvature and its isoperimetric deficit for higher-dimensional conformal Euclidean metrics, Calc. Var. PDEs, 38 (2010), nos 1-2, 1-27, MR 2610523, Zbl 1198.53009. 
[64] S. Paneitz, A quartic conformally covariant differential operator for arbitrary pseudo-Riemannian manifolds, SIGMA Symmetry Integrability Geom. Methods Appl. 4 (2008), Paper 036, 3 pp., MR 2393291, Zbl 1145.53053.

[65] M. Ould Ahmedou, Conformal deformations of Riemannian metrics via "critical point theory at infinity": the conformally flat case with umbilic boundary. (English summary) Noncompact problems at the intersection of geometry, analysis, and topology, 1-17, Contemp. Math., 350, Amer. Math. Soc., Providence, RI, 2004, MR 2082387, Zbl 1080.58020.

[66] R. Schoen, Conformal deformation of a Riemannian metric to constant scalar curvature, J. Differential Geom. 20 (1984), no. 2, 479-495, MR 0788292, Zbl 0576.53028 .

[67] R. Schoen \& D. Zhang, Prescribing scalar curvature on the $n$-sphere, Cal. Var. PDEs 4 (1996), MR 1379191, Zbl 0843.53037.

[68] H. Schwetlick \& M. Struwe, Convergence of the Yamabe flow for "large" energies, J. Reine. Angew. Math. 562 (2003), 59-100, MR 2011332, Zbl 1079.53100.

\section{Mathematisches Institut DER Justus-Liebig-Universität Giessen ARNDTSTRASSE 2 D-35392 GIESSEN Germany}

E-mail address: martin.g.mayer@math.uni-giessen.de

$$
\begin{array}{r}
\text { Fachbereich Mathematik } \\
\text { Der Eberhard-Karls-Universität TüBingen } \\
\text { Auf Der Morgenstelle } 10 \\
\text { D-72076 Tübingen } \\
\text { Germany }
\end{array}
$$

E-mail address: ndiaye@everest.mathematik.uni-tuebingen.de

AND

Mathematisches Institut

DER Justus-Liebig-Universität Giessen

ARndTStrasse 2

D-35392 GIESSEN

Germany

E-mail address: ndiaye@math.uni-giessen.de 